\title{
Constrained characteristic functions, multivariable interpolation, and invariant subspaces
}

\author{
Jian $\mathrm{Hu}^{1 *}$ (D), Maofa Wang ${ }^{2}$ and Wei Wang
}

\section{"Correspondence:}

jhu.math@whu.edu.cn

${ }^{1}$ School of Mathematics and

Statistics, Hubei Normal University, Huangshi, China

Full list of author information is

available at the end of the article

\section{Springer}

\begin{abstract}
In this paper, we present a functional model theorem for completely non-coisometric $n$-tuples of operators in the noncommutative variety $\mathcal{V}_{f, \varphi, \mathcal{I}}(\mathcal{H})$ in terms of constrained characteristic functions. As an application, we prove that the constrained characteristic function is a complete unitary invariant for this class of elements, which can be viewed as the noncommutative analogue of the classical Sz.-Nagy-Foiaş functional model for completely nonunitary contractions. On the other hand, we provide a Sarason-type commutant lifting theorem. Applying this result, we solve the Nevanlinna-Pick-type interpolation problem in our setting. Moreover, we also obtain a Beurling-type characterization of the joint invariant subspaces under the operators $B_{1}, \ldots, B_{n}$, where the $n$-tuple $\left(B_{1}, \ldots, B_{n}\right)$ is the universal model associated with the abstract noncommutative variety $\mathcal{V}_{f, \varphi, \mathcal{I}}$.
\end{abstract}

MSC: Primary 46L52; 47A45; secondary 46L07; 46T25

Keywords: Noncommutative Poisson transform; Characteristic function; Universal model; Interpolation

\section{Introduction}

In the last fifty years, the study of the closed operator unit ball

$$
[B(\mathcal{H})]_{1}^{-}:=\left\{T \in B(\mathcal{H}):\left\|T T^{*}\right\|^{\frac{1}{2}} \leq 1\right\}
$$

has generated the celebrated Sz.-Nagy-Foiaş theory of contractions on Hilbert spaces. This research has evolved into a well-developed theory, which plays an important role in modern functional analysis. In 1963, Sz.-Nagy and Foiaş obtained an effective $H^{\infty}$ functional calculus for completely nonunitary contractions on Hilbert spaces based on the existence of a unitary dilation of a contraction $T$ (see [33]). An important application of this functional calculus to the theory of contraction semigroups has also been given in Foiaş [5]. Moreover, the characteristic function of a contraction $T$ appears as the operatorvalued analytic function corresponding to a certain orthogonal projection in the space of the minimal unitary dilation of $T$. This yields a functional model for $T$, which is a useful tool for analyzing the structure of contractions.

(c) The Author(s) 2020. This article is licensed under a Creative Commons Attribution 4.0 International License, which permits use, sharing, adaptation, distribution and reproduction in any medium or format, as long as you give appropriate credit to the original author(s) and the source, provide a link to the Creative Commons licence, and indicate if changes were made. The images or other third party material in this article are included in the article's Creative Commons licence, unless indicated otherwise in a credit line to the material. If material is not included in the article's Creative Commons licence and your intended use is not permitted by statutory regulation or exceeds the permitted use, you will need to obtain permission directly from the copyright holder. To view a copy of this licence, visit http://creativecommons.org/licenses/by/4.0/ 
In the multivariable case, the study of the closed operator unit $n$-ball

$$
\left[B(\mathcal{H})^{n}\right]_{1}^{-}:=\left\{\left(T_{1}, \ldots, T_{n}\right) \in B(\mathcal{H})^{n}:\left\|T_{1} T_{1}^{*}+\cdots+T_{n} T_{n}^{*}\right\|^{\frac{1}{2}} \leq 1\right\}
$$

has generated a noncommutative analogue of Sz.-Nagy-Foiaş theory (see [2-4, 6-8], and more recently $[1,11,34])$. In particular, Popescu developed a theory of holomorphic functions in several noncommuting variables and provided a framework for the study of arbitrary $n$-tuples of operators. A free analytic functional calculus was introduced and studied in connection with Hausdorff derivations, noncommutative Cauchy and Poisson transforms, and von Neumann inequalities (see [15, 16, 18, 20-23, 26, 29, 30]). Moreover, we remark the work of Helton, McCullough, and Vinnikov on symmetric noncommutative polynomials (see $[9,10]$ ). We should also remark that, in recent years, many results concerning the theory of row contractions were extended by Muhly and Solel ([12-14]) to representations of tensor algebras over $C^{*}$-correspondences and Hardy algebras.

In [28], Popescu developed an operator model theory for pure $n$-tuples of operators in noncommutative domains $\mathbb{D}_{f, \varphi}(\mathcal{H}) \subset B(\mathcal{H})^{n}$ generated by positive regular free holomorphic functions $f$ and certain classes of $n$-tuples $\varphi=\left(\varphi_{1}, \ldots, \varphi_{n}\right)$ of formal power series in noncommutative indeterminates $Z_{1}, \ldots, Z_{n}$. An important role in his study was played by noncommutative Poisson transforms. Using these transforms, he proved that each abstract noncommutative domain $\mathbb{D}_{f, \varphi}$ has a universal model $\left(M_{Z_{1}}, \ldots, M_{Z_{n}}\right)$. Unlike the case of the ball $\left[B(\mathcal{H})^{n}\right]_{1}^{-}$, the operators $M_{Z_{1}}, \ldots, M_{Z_{n}}$ are not isometries and do not have orthogonal ranges in general, which leads to considerable technical difficulties in developing an operator model theory. Moreover, notice that the study of $\mathbb{D}_{f, \varphi}(\mathcal{H})$ is closely related to the study of the operators $M_{Z_{1}}, \ldots, M_{Z_{n}}$, their joint invariant subspaces, and the representations of the algebras they generate: the noncommutative domain algebra $\mathcal{A}\left(\mathbb{D}_{f, \varphi}\right)$, the noncommutative Hardy algebra $H^{\infty}\left(\mathbb{D}_{f, \varphi}\right)$, and the $C^{*}$-algebra $C^{*}\left(M_{Z_{1}}, \ldots, M_{Z_{n}}\right)$. Indeed, this noncommutative domain $\mathbb{D}_{f, \varphi}(\mathcal{H})$ has been studied in several particular cases. According to [22, 24] and [33], if $f=Z$ and $\varphi=Z$, then the corresponding domain $\mathbb{D}_{f, \varphi}(\mathcal{H})$ coincides with the closed operator unit ball $[B(\mathcal{H})]_{1}^{-}$, the study of which has generated Sz.-Nagy-Foiaş theory of contractions. If $f=Z_{1}+\cdots+Z_{n}$ and $\varphi=\left(Z_{1}, \ldots, Z_{n}\right)$, then the corresponding domain $\mathbb{D}_{f, \varphi}(\mathcal{H})$ coincides with the closed operator unit $n$-ball $\left[B(\mathcal{H})^{n}\right]_{1}^{-}$, the study of which has generated a free analogue of Sz.-Nagy-Foiaş theory. In particular, if $\varphi=\left(Z_{1}, \ldots, Z_{n}\right)$, then the corresponding domain $\mathbb{D}_{f, \varphi}(\mathcal{H})$ coincides with the noncommutative Reinhardt domain $\mathcal{D}_{f}(\mathcal{H})$, which was first studied by Popescu [24].

In this paper, we continue the research line of Popescu to develop an operator model theory for completely non-coisometric $n$-tuples of operators in noncommutative varieties $\mathcal{V}_{f, \varphi, \mathcal{I}}(\mathcal{H})$. To present our results, we need some notation. Let $\mathbf{S}\left[Z_{1}, \ldots, Z_{n}\right]$ be the algebra of all formal power series in noncommutative indeterminates $Z_{1}, \ldots, Z_{n}$ and complex coefficients. We denote by $\mathbb{F}_{n}^{+}$the unital free semigroup on $n$ generators $g_{1}, \ldots, g_{n}$ and the identity $g_{0}$. The length of $\alpha \in \mathbb{F}_{n}^{+}$is defined by $|\alpha|:=0$ if $\alpha=g_{0}$ and $|\alpha|:=k$ if $\alpha=g_{i_{1}} \cdots g_{i_{k}}$, where $i_{1}, \ldots, i_{k} \in\{1, \ldots, n\}$. We set $Z_{\alpha}:=Z_{i_{1}} \ldots Z_{i_{k}}$ and $Z_{g_{0}}:=I$. If $f \in \mathbf{S}\left[Z_{1}, \ldots, Z_{n}\right]$ has the representation $f:=\sum_{\alpha \in \mathbb{F}_{n}^{+}} a_{\alpha} Z_{\alpha}$ and the coefficients $a_{\alpha} \in \mathbb{C}$ satisfy the conditions

$$
r(f)^{-1}:=\limsup _{k \rightarrow \infty}\left(\sum_{|\alpha|=k}\left|a_{\alpha}\right|^{2}\right)^{\frac{1}{2 k}}<\infty,
$$


$a_{\alpha} \geq 0$ for any $\alpha \in \mathbb{F}_{n}^{+}, a_{g_{0}}=0$, and $a_{g_{i}}>0, i=1, \ldots, n$, we say that $f$ is a positive regular free holomorphic function. The number $r(f)$ is called the radius of convergence of $f$.

Denote by $\mathcal{M}_{f}$ the set of all $n$-tuples $\varphi=\left(\varphi_{1}, \ldots, \varphi_{n}\right)$ of formal power series $\varphi_{i} \in$ $\mathbf{S}\left[Z_{1}, \ldots, Z_{n}\right]$ with the model property (see Sect. 2$) . \mathcal{H}$ is a Hilbert space and $B(\mathcal{H})$ is the algebra of all bounded linear operators on $\mathcal{H}$. If $X=\left(X_{1}, \ldots, X_{n}\right) \in B(\mathcal{H})^{n}$, we denote $X_{\alpha}:=X_{i_{1}} \cdots X_{i_{k}}$ if $\alpha=g_{i_{1}} \cdots g_{i_{k}} \in \mathbb{F}_{n}^{+}$, and $X_{g_{0}}:=I_{\mathcal{H}}$. We introduce the noncommutative domain $\mathbb{D}_{f, \varphi}(\mathcal{H})$ associated with $f, \varphi \in \mathcal{M}_{f}$ and a Hilbert space $\mathcal{H}$ and defined by

$$
\mathbb{D}_{f, \varphi}(\mathcal{H}):=\left\{X \in B(\mathcal{H})^{n}: \psi(\varphi(X))=X \text { and } \sum_{|\alpha| \geq 1} a_{\alpha}[\varphi(X)]_{\alpha}[\varphi(X)]_{\alpha}^{*} \leq I_{\mathcal{H}}\right\}
$$

where $\psi:=\left(\psi_{1}, \ldots, \psi_{n}\right)$ is the inverse of $\varphi$ with respect to composition of formal power series, and the evaluations are well defined (see Sect. 2). We refer to $\mathbb{D}_{f, \varphi}:=\left\{\mathbb{D}_{f, \varphi}(\mathcal{H})\right.$ : $\mathcal{H}$ is a Hilbert space $\}$ as the abstract noncommutative domain, and to $\mathbb{D}_{f, \varphi}(\mathcal{H})$ as its representation on the Hilbert space $\mathcal{H}$. We associate with each $\mathbb{D}_{f, \varphi}$ a Hilbert space $\mathbb{H}_{f}^{2}(\varphi)$ of formal power series in $\mathbf{S}\left[Z_{1}, \ldots, Z_{n}\right]$ with the property that the indeterminates $Z_{1}, \ldots, Z_{n}$ are in the Hilbert space $\mathbb{H}_{f}^{2}(\varphi)$ and each left multiplication operator $M_{Z_{i}}: \mathbb{H}_{f}^{2}(\varphi) \rightarrow \mathbb{H}_{f}^{2}(\varphi)$ defined by

$$
M_{Z_{i}} \zeta:=Z_{i} \zeta, \quad \zeta \in \mathbb{H}_{f}^{2}(\varphi)
$$

is a bounded multiplier of $\mathbb{H}_{f}^{2}(\varphi)$. Similarly, each right multiplication operator $R_{Z_{i}}$ : $\mathbb{H}_{f}^{2}(\varphi) \rightarrow \mathbb{H}_{f}^{2}(\varphi)$ defined by

$$
R_{Z_{i}} \zeta:=\zeta Z_{i}, \quad \zeta \in \mathbb{H}_{f}^{2}(\varphi)
$$

is also a bounded multiplier of $\mathbb{H}_{f}^{2}(\varphi)$.

Let $\mathcal{I} \neq H^{\infty}\left(\mathbb{D}_{f, \varphi}\right)$ be a WOT-closed two-sided ideal of the noncommutative Hardy algebra $H^{\infty}\left(\mathbb{D}_{f, \varphi}\right)$, where $H^{\infty}\left(\mathbb{D}_{f, \varphi}\right)$ is the WOT-closure of all noncommutative polynomials in $M_{Z_{1}}, \ldots, M_{Z_{n}}$ and the identity. Now we define the noncommutative variety

$$
\mathcal{V}_{f, \varphi, \mathcal{I}}(\mathcal{H}):=\left\{\left(X_{1}, \ldots, X_{n}\right) \in \mathbb{D}_{f, \varphi}(\mathcal{H}): \omega\left(X_{1}, \ldots, X_{n}\right)=0 \text { for any } \omega \in \mathcal{I}\right\}
$$

Denote by $H^{\infty}\left(\mathcal{V}_{f, \varphi, \mathcal{I}}\right)$ the WOT-closed algebra generated by the constrained weighted shifts $B_{i}:=\left.P_{\mathcal{N}_{f, \varphi, \mathcal{I}}} M_{Z_{i}}\right|_{\mathcal{N}_{f, \varphi, \mathcal{I}}}$ for $i=1, \ldots, n$ and the identity, where

$$
\mathcal{N}_{f, \varphi, \mathcal{I}}:=\mathbb{H}_{f}^{2}(\varphi) \ominus \mathcal{M}_{f, \varphi, \mathcal{I}} \quad \text { and } \quad \mathcal{M}_{f, \varphi, \mathcal{I}}:=\overline{\mathcal{I} \mathbb{H}_{f}^{2}(\varphi)}
$$

Similarly, denote by $R^{\infty}\left(\mathcal{V}_{f, \varphi, \mathcal{I}}\right)$ the WOT-closed algebra generated by the constrained weighted shifts $C_{i}:=\left.P_{\mathcal{N}_{f, \varphi, \mathcal{I}}} R_{Z_{i}}\right|_{\mathcal{N}_{f, \varphi, \mathcal{I}}}$ for $i=1, \ldots, n$ and the identity.

In Sect. 2, we collect some notation and preliminaries which are needed in the sequel. In Sect. 3, we obtain a factorization result for the constrained characteristic function, namely

$$
I_{\mathcal{N}_{f, \varphi, \mathcal{I}} \otimes \mathcal{D}_{C f, \varphi, T}}-\Theta_{f, \varphi, T}^{(\mathcal{I})}\left(\Theta_{f, \varphi, T}^{(\mathcal{I})}\right)^{*}=K_{f, \varphi, T}^{(\mathcal{I})}\left(K_{f, \varphi, T}^{(\mathcal{I})}\right)^{*},
$$

where $\Theta_{f, \varphi, T}^{(\mathcal{I})}$ is the constrained characteristic function and $K_{f, \varphi, T}^{(\mathcal{I})}$ is the corresponding constrained Poisson kernel. Moreover, we present a functional model theorem for completely 
non-coisometric $n$-tuples of operators in the noncommutative variety $\mathcal{V}_{f, \varphi, \mathcal{I}}(\mathcal{H})$ in terms of constrained characteristic functions. Applying this result, we prove that the constrained characteristic function is a complete unitary invariant for this class of elements. Indeed, this result can be viewed as the noncommutative analogue of the classical Sz.-Nagy-Foiaş functional model for completely nonunitary contractions.

In Sect. 4, we prove a Sarason-type commutant lifting theorem. As an application, we obtain the Nevanlinna-Pick-type interpolation result in our setting. We show that if $\lambda_{1}, \ldots, \lambda_{k}$ are $k$ distinct points in the strict noncommutative variety $\mathcal{V}_{f, \varphi, \mathcal{I}}^{<}(\mathbb{C})$ and $A_{1}, \ldots, A_{k} \in B(\mathcal{K})$, then there exists $\Phi\left(C_{1}, \ldots, C_{n}\right) \in R^{\infty}\left(\mathcal{V}_{f, \varphi, \mathcal{I}}\right) \bar{\otimes} B(\mathcal{K})$ such that

$$
\left\|\Phi\left(C_{1}, \ldots, C_{n}\right)\right\| \leq 1 \quad \text { and } \quad \Phi\left(\lambda_{j}\right)=A_{j}, \quad j=1, \ldots, k
$$

if and only if the operator matrix

$$
\left[K_{f, \varphi}\left(\lambda_{i}, \lambda_{j}\right)\left(I_{\mathcal{K}}-A_{i} A_{j}^{*}\right)\right]_{k \times k}
$$

is positive semidefinite, where

$$
K_{f, \varphi}\left(\lambda_{i}, \lambda_{j}\right):=\frac{\sqrt{1-\sum_{|\alpha| \geq 1} a_{\alpha}\left|\varphi_{\alpha}\left(\lambda_{i}\right)\right|^{2}} \sqrt{1-\sum_{|\alpha| \geq 1} a_{\alpha}\left|\varphi_{\alpha}\left(\lambda_{j}\right)\right|^{2}}}{1-\sum_{|\alpha| \geq 1} a_{\alpha}\left[\varphi\left(\lambda_{i}\right)\right]_{\alpha}\left[\overline{\varphi\left(\lambda_{j}\right)}\right]_{\alpha}} .
$$

Moreover, we provide a Beurling-type characterization of the joint invariant subspaces under the constrained weighted shifts $B_{1}, \ldots, B_{n}$. More precisely, a subspace $\mathcal{M} \subseteq \mathcal{N}_{f, \varphi, \mathcal{I}} \otimes$ $\mathcal{K}$ is invariant under $B_{i} \otimes I_{\mathcal{K}}, i=1, \ldots, n$, if and only if there are a Hilbert space $\mathcal{G}$ and an inner multi-analytic operator

$$
\Phi: \mathcal{N}_{f, \varphi, \mathcal{I}} \otimes \mathcal{G} \rightarrow \mathcal{N}_{f, \varphi, \mathcal{I}} \otimes \mathcal{K}
$$

with respect to the constrained weighted shifts $B_{1}, \ldots, B_{n}$ such that

$$
\mathcal{M}=\Phi\left[\mathcal{N}_{f, \varphi, \mathcal{I}} \otimes \mathcal{G}\right]
$$

\section{Preliminaries}

In this section we collect some notation and preliminaries which are needed in the sequel. For more information, we refer to [24, 27] and [28].

\subsection{Weighted Fock space}

Let $f:=\sum_{\alpha \in \mathbb{F}_{n}^{+}} a_{\alpha} Z_{\alpha}, a_{\alpha} \in \mathbb{C}$, be a positive regular free holomorphic function. Define the noncommutative domain

$$
\mathcal{D}_{f}(\mathcal{H}):=\left\{\left(X_{1}, \ldots, X_{n}\right) \in B(\mathcal{H})^{n}: \sum_{|\alpha| \geq 1} a_{\alpha} X_{\alpha} X_{\alpha}^{*} \leq I_{\mathcal{H}}\right\},
$$

where the convergence of the series is in the weak operator topology. Define the strict noncommutative domain

$$
\mathcal{D}_{f,<}(\mathcal{H}):=\left\{\left(X_{1}, \ldots, X_{n}\right) \in B(\mathcal{H})^{n}:\left\|\sum_{|\alpha| \geq 1} a_{\alpha} X_{\alpha} X_{\alpha}^{*}\right\|<1\right\},
$$


where the convergence is in the weak operator topology. Now, we define

$$
b_{g_{0}}=1 \quad \text { and } \quad b_{\alpha}=\sum_{j=1}^{|\alpha|} \sum_{\substack{\gamma_{1} \cdots, \gamma_{j}=\alpha \\\left|\gamma_{1}\right| \geq 1, \ldots,\left|\gamma_{j}\right| \geq 1}} a_{\gamma_{1}} \cdots a_{\gamma_{j}} \quad \text { if }|\alpha| \geq 1 .
$$

We introduce an inner product on the algebra of noncommutative polynomials $\mathbb{C}\left[Z_{1}, \ldots, Z_{n}\right]$ by setting

$$
\left\langle Z_{\alpha}, Z_{\beta}\right\rangle_{f}:=\frac{1}{b_{\alpha}} \delta_{\alpha \beta}, \quad \alpha, \beta \in \mathbb{F}_{n}^{+} .
$$

Let $\mathcal{F}_{f}^{2}$ be the completion of $\mathbb{C}\left[Z_{1}, \ldots, Z_{n}\right]$ in this inner product. Notice that the elements of $\mathcal{F}_{f}^{2}$ are formal power series $\zeta \in \mathbf{S}\left[Z_{1}, \ldots, Z_{n}\right]$ of the form $\zeta=\sum_{\alpha \in \mathbb{F}_{n}^{+}} c_{\alpha} Z_{\alpha}$, where

$$
\|\zeta\|_{f}^{2}:=\sum_{\alpha \in \mathbb{F}_{n}^{+}}\left|c_{\alpha}\right|^{2} \frac{1}{b_{\alpha}}<\infty
$$

Indeed, $\mathcal{F}_{f}^{2}$ is a weighted Fock space on $n$ generators. For each $i=1, \ldots, n$, we define the left multiplication operator $V_{i}: \mathcal{F}_{f}^{2} \rightarrow \mathcal{F}_{f}^{2}$ by setting $V_{i} \zeta:=Z_{i} \zeta$. Notice that $\left(V_{1}, \ldots, V_{n}\right)$ is in the noncommutative domain $\mathcal{D}_{f}\left(\mathcal{F}_{f}^{2}\right)$, and

$$
I_{\mathcal{F}_{f}^{2}}-\sum_{|\alpha| \geq 1} a_{\alpha} V_{\alpha} V_{\alpha}^{*}=P_{\mathbb{C}}
$$

where $P_{\mathbb{C}}$ is the orthogonal projection from $\mathcal{F}_{f}^{2}$ onto $\mathbb{C}$.

Let $\mathcal{F}_{f}^{\infty}$ be the set of all $\zeta \in \mathcal{F}_{f}^{2}$ with the property that

$$
\|\zeta\|_{\infty}:=\sup \left\{\|\zeta p\|_{f}: p \in \mathbb{C}\left[Z_{1}, \ldots, Z_{n}\right],\|p\|_{f} \leq 1\right\}<\infty .
$$

Notice that $\mathcal{F}_{f}^{\infty}$ is a Banach algebra with respect to the norm $\|\cdot\|_{\infty}$. Let $\zeta=\sum_{\beta \in \mathbb{F}_{n}^{+}} c_{\beta} Z_{\beta}$ be a formal power series with the property that $\sum_{\beta \in \mathbb{F}_{n}^{+}}\left|c_{\beta}\right|^{2} \frac{1}{b_{\beta}}<\infty$, where the coefficients $b_{\beta}, \beta \in \mathbb{F}_{n}^{+}$, are given by relation (2.1). One can see that $\sum_{\beta \in \mathbb{F}_{n}^{+}} c_{\beta} V_{\beta}(p) \in \mathcal{F}_{f}^{2}$ for any $p \in$ $\mathbb{C}\left[Z_{1}, \ldots, Z_{n}\right]$. Moreover, $\zeta \in \mathcal{F}_{f}^{\infty}$ if and only if

$$
\sup _{p \in \mathbb{C}\left[Z_{1}, \ldots, Z_{n}\right],\|p\|_{f} \leq 1}\left\|\sum_{\beta \in \mathbb{F}_{n}^{+}} c_{\beta} V_{\beta}(p)\right\|_{f}<\infty .
$$

In this case, there is a unique bounded operator acting on $\mathcal{F}_{f}^{2}$, which we denote by $\zeta\left(V_{1}, \ldots, V_{n}\right)$, such that

$$
\zeta\left(V_{1}, \ldots, V_{n}\right) p=\sum_{\beta \in \mathbb{F}_{n}^{+}} c_{\beta} V_{\beta}(p) \text { for any } p \in \mathbb{C}\left[Z_{1}, \ldots, Z_{n}\right]
$$

We call the series $\sum_{\beta \in \mathbb{F}_{n}^{+}} c_{\beta} V_{\beta}$ the Fourier representation of $\zeta\left(V_{1}, \ldots, V_{n}\right)$. The set of all operators $\varphi\left(V_{1}, \ldots, V_{n}\right) \in B\left(\mathcal{F}_{f}^{2}\right)$ satisfying the above-mentioned properties is denoted by $F^{\infty}\left(\mathcal{D}_{f}\right)$ 
We consider the full Fock space of $H_{n}$ defined by

$$
F^{2}\left(H_{n}\right):=\mathbb{C} 1 \oplus \bigoplus_{m \geq 1} H_{n}^{\otimes m}
$$

where $H_{n}^{\otimes m}$ is the Hilbert tensor product of $m$ copies of $H_{n}$. We denote $e_{\alpha}:=e_{i_{1}} \otimes \cdots \otimes e_{i_{k}}$ if $\alpha=g_{i_{1}} \cdots g_{i_{k}}$, where $i_{1}, \ldots, i_{k} \in\{1, \ldots, n\}$, and $e_{g_{0}}:=1$. Consider $\Omega: F^{2}\left(H_{n}\right) \rightarrow \mathcal{F}_{f}^{2}$ to be the unitary operator defined by $\Omega\left(e_{\alpha}\right):=\sqrt{b_{\alpha}} Z_{\alpha}, \alpha \in \mathbb{F}_{n}^{+}$, where the coefficients $b_{\alpha}$ are given by relation (2.1). We remark that $\Omega^{-1} V_{i} \Omega=W_{i}, i=1, \ldots, n$, where $\left(W_{1}, \ldots, W_{n}\right)$ is the $n$-tuple of weighted shifts on $F^{2}\left(H_{n}\right)$, which was introduced in [24]. Using the results from [24], we know that $F^{\infty}\left(\mathcal{D}_{f}\right)$ is the WOT-closure (resp. SOT-closure, $w^{*}$-closure) of all polynomials in $V_{1}, \ldots, V_{n}$ and the identity. The noncommutative domain algebra $\mathcal{A}\left(\mathcal{D}_{f}\right)$ is the norm-closure of all polynomials in $V_{1}, \ldots, V_{n}$ and the identity.

\subsection{Noncommutative domain}

We say that an $n$-tuple $p=\left(p_{1}, \ldots, p_{n}\right)$ of polynomials is invertible with respect to composition if there exists an $n$-tuple $q=\left(q_{1}, \ldots, q_{n}\right)$ of polynomials such that $p \circ q=q \circ p=i d$. In this case, we say that $p$ has property $(\mathcal{A})$. In what follows, we provide an example. If

$$
\begin{aligned}
& p_{1}=a_{1} Z_{1}+a_{2} Z_{2}+a_{3} Z_{3} Z_{2}, \\
& p_{2}=b_{2} Z_{2}+b_{3} Z_{3}^{2} \quad\left(a_{1} b_{2} c_{3} \neq 0\right), \\
& p_{3}=c_{3} Z_{3},
\end{aligned}
$$

then $p=\left(p_{1}, p_{2}, p_{3}\right)$ is invertible with respect to composition, i.e., there exists $q=\left(q_{1}, q_{2}, q_{3}\right)$ such that $p \circ q=q \circ p=i d$, where

$$
\begin{aligned}
& q_{1}=\frac{1}{a_{1}} Z_{1}-\frac{a_{2}}{a_{1} b_{2}} Z_{2}-\frac{a_{3}}{a_{1} b_{2} c_{3}} Z_{3} Z_{2}+\frac{a_{2} b_{3}}{a_{1} b_{2} c_{3}^{2}} Z_{3}^{2}+\frac{a_{3} b_{3}}{a_{1} b_{2} c_{3}^{3}} Z_{3}^{3}, \\
& q_{2}=\frac{1}{b_{2}} Z_{2}-\frac{b_{3}}{b_{2} c_{3}^{2}} Z_{3}^{2}, \\
& q_{3}=\frac{1}{c_{3}} Z_{3} .
\end{aligned}
$$

This shows that $p$ has property $(\mathcal{A})$.

Let $f:=\sum_{\alpha \in \mathbb{F}_{n}^{+}} a_{\alpha} Z_{\alpha}$ be a positive regular free holomorphic function, and let $p=$ $\left(p_{1}, \ldots, p_{n}\right)$ be an $n$-tuple of noncommutative polynomials with property $(\mathcal{A})$. We introduce an inner product by setting

$$
\left\langle p_{\alpha}, p_{\beta}\right\rangle_{f, p}:=\frac{1}{b_{\alpha}} \delta_{\alpha \beta}, \quad \alpha, \beta \in \mathbb{F}_{n}^{+}
$$

Let $\mathbb{H}_{f}^{2}(p)$ be the completion of the linear space $\bigvee\left\{p_{\alpha}\right\}_{\alpha \in \mathbb{F}_{n}^{+}}$with respect to this inner product.

Consider an $n$-tuple of formal power series $\varphi=\left(\varphi_{1}, \ldots, \varphi_{n}\right)$ in indeterminates $Z_{1}, \ldots, Z_{n}$ with the property that the Jacobian

$$
\operatorname{det} J_{\varphi}(0):=\operatorname{det}\left[\lambda_{i j}\right]_{i, j=1}^{n} \neq 0
$$


where

$$
\varphi_{i}\left(Z_{1}, \ldots, Z_{n}\right)=a_{0}^{(i)} I+\sum_{p=1}^{n} a_{p}^{(i)} Z_{p}+\sum_{|\alpha| \geq 2} a_{\alpha}^{(i)} Z_{\alpha}, \lambda_{i j}=a_{j}^{(i)}
$$

and $i, j=1, \ldots, n$. Due to Theorem 1.2 from [25], the set $\left\{\varphi_{\alpha}\right\}_{\alpha \in \mathbb{F}_{n}^{+}}$(where $\varphi_{0}:=I$ ) is linearly independent in $\mathbf{S}\left[Z_{1}, \ldots, Z_{n}\right]$. We introduce an inner product on the linear span of $\left\{\varphi_{\alpha}\right\}_{\alpha \in \mathbb{F}_{n}^{+}}$ by setting

$$
\left\langle\varphi_{\alpha}, \varphi_{\beta}\right\rangle_{f, \varphi}:=\frac{1}{b_{\alpha}} \delta_{\alpha \beta}, \quad \alpha, \beta \in \mathbb{F}_{n}^{+},
$$

where the coefficients $b_{\alpha}, \alpha \in \mathbb{F}_{n}^{+}$, are given by relation (2.1). Let $\mathbb{H}_{f}^{2}(\varphi)$ be the completion of the linear space $\bigvee\left\{\varphi_{\alpha}\right\}_{\alpha \in \mathbb{F}_{n}^{+}}$with respect to this inner product. Assume now that $\varphi(0)=0$. Theorem 1.3 from [25] shows that $\varphi$ is not a right zero divisor with respect to composition, i.e., there is no nonzero power series $\chi$ in $\mathbf{S}\left[Z_{1}, \ldots, Z_{n}\right]$ such that $\chi \circ \varphi=0$. Consequently, the elements of $\mathbb{H}_{f}^{2}(\varphi)$ can be seen as a formal power series in $\mathbf{S}\left[Z_{1}, \ldots, Z_{n}\right]$ of the form $\sum_{\alpha \in \mathbb{F}_{n}^{+}} c_{\alpha} \varphi_{\alpha}$, where $\sum_{\alpha \in \mathbb{F}_{n}^{+}} \frac{1}{b_{\alpha}}\left|c_{\alpha}\right|^{2}<\infty$.

To introduce the class of $n$-tuples of formal power series with property $(\mathcal{S})$, we need some preliminaries. Let $\chi=\sum_{k=0}^{\infty} \sum_{|\alpha|=k} c_{\alpha} Z_{\alpha}$ be a formal power series in indeterminates $Z_{1}, \ldots, Z_{n}$. We denote by $\mathcal{C}_{\chi}(\mathcal{H})\left(\right.$ resp. $\left.\mathcal{C}_{\chi}^{\text {SOT }}(\mathcal{H})\right)$ the set of all $Y:=\left(Y_{1}, \ldots, Y_{n}\right) \in B(\mathcal{H})^{n}$ such that the series $\chi\left(Y_{1}, \ldots, Y_{n}\right):=\sum_{k=0}^{\infty} \sum_{|\alpha|=k} c_{\alpha} Y_{\alpha}$ is norm (resp. SOT) convergent. These sets are called sets of norm (resp. SOT) convergence for the power series $\chi$. We also introduce the $\operatorname{set} \mathcal{C}_{\chi}^{\mathrm{rad}}(\mathcal{H})$ of all $Y:=\left(Y_{1}, \ldots, Y_{n}\right) \in B(\mathcal{H})^{n}$ such that there exists $\delta \in(0,1)$ with the property that $r Y \in \mathcal{C}_{\chi}(\mathcal{H})$ for any $r \in(\delta, 1)$ and

$$
\widehat{\chi}\left(Y_{1}, \ldots, Y_{n}\right):=\text { SOT }-\lim _{r \rightarrow 1} \sum_{k=0}^{\infty} \sum_{|\alpha|=k} c_{\alpha} r^{|\alpha|} Y_{\alpha}
$$

exists.

Definition 2.1 (see [28]) Let $\varphi=\left(\varphi_{1}, \ldots, \varphi_{n}\right)$ be an $n$-tuple of formal power series in $Z_{1}, \ldots, Z_{n}$ such that $\varphi(0)=0$. We say that $\varphi$ has property $(\mathcal{S})$ if the following conditions hold:

$\left(\mathcal{S}_{1}\right)$ The radius of convergence of $\varphi$, i.e., $r(\varphi):=\min _{i=1, \ldots, n} r\left(\varphi_{i}\right)$, is strictly positive and det $J_{\varphi}(0) \neq 0$.

$\left(\mathcal{S}_{2}\right)$ The indeterminates $Z_{1}, \ldots, Z_{n}$ are in the Hilbert space $\mathbb{H}_{f}^{2}(\varphi)$ and each multiplication operator $M_{Z_{i}}: \mathbb{H}_{f}^{2}(\varphi) \rightarrow \mathbb{H}_{f}^{2}(\varphi)$ defined by

$$
M_{Z_{i}} \zeta:=Z_{i} \zeta, \quad \zeta \in \mathbb{H}_{f}^{2}(\varphi)
$$

is a bounded multiplier of $\mathbb{H}_{f}^{2}(\varphi)$.

$\left(\mathcal{S}_{3}\right)$ The multiplication operators $M_{\varphi_{j}}: \mathbb{H}_{f}^{2}(\varphi) \rightarrow \mathbb{H}_{f}^{2}(\varphi), M_{\varphi_{j}} \chi=\varphi_{j} \chi$, satisfy the equations

$$
M_{\varphi_{j}}=\varphi_{j}\left(M_{Z_{1}}, \ldots, M_{Z_{n}}\right), \quad j=1, \ldots, n,
$$

where $\left(M_{Z_{1}}, \ldots, M_{Z_{n}}\right)$ is either in the convergence $\operatorname{set} \mathcal{C}_{\varphi}^{\text {SOT }}\left(\mathbb{H}_{f}^{2}(\varphi)\right)$ or $\mathcal{C}_{\varphi}^{\text {rad }}\left(\mathbb{H}_{f}^{2}(\varphi)\right)$. 
Let $U: \mathbb{H}_{f}^{2}(\varphi) \rightarrow \mathcal{F}_{f}^{2}$ be the unitary operator defined by $U\left(\varphi_{\alpha}\right):=Z_{\alpha}, \alpha \in \mathbb{F}_{n}^{+}$. According to the proof of Lemma 1.2 from [28], we have

$$
M_{\varphi_{i}}=U^{-1} V_{i} U, \quad i=1, \ldots, n .
$$

Throughout this paper, unless otherwise specified, we assume that $\varphi=\left(\varphi_{1}, \ldots, \varphi_{n}\right)$ is either an $n$-tuple of noncommutative polynomials with property $(\mathcal{A})$ or an $n$-tuple of formal power series with $\varphi(0)=0$ and property $(\mathcal{S})$. In this case, we say that $\varphi$ has the model property.

Definition 2.2 (see $[25,28])$ Let $\varphi=\left(\varphi_{1}, \ldots, \varphi_{n}\right)$ be an $n$-tuple of formal power series with model property, and let $\psi=\left(\psi_{1}, \ldots, \psi_{n}\right)$ be the $n$-tuple of power series which is the inverse of $\varphi=\left(\varphi_{1}, \ldots, \varphi_{n}\right)$ with respect to composition. Assume that $\psi_{i}$ has the representation

$$
\psi_{i}=\sum_{k=0}^{\infty} \sum_{\alpha \in \mathbb{F}_{n}^{+},|\alpha|=k} c_{\alpha}^{(i)} Z_{\alpha} \quad \text { for } i=1, \ldots, n
$$

where the sequence $\left\{c_{\alpha}^{(i)}\right\}_{\alpha \in \mathbb{F}_{n}^{+}}$is uniquely determined by the condition $\psi \circ \varphi=i d$. We say that an $n$-tuple of operators $X=\left(X_{1}, \ldots, X_{n}\right) \in B(\mathcal{H})^{n}$ satisfies the equation $\psi(\varphi(X))=X$ in either one of the following two cases:

(a) $X \in \mathcal{C}_{\varphi}^{\mathrm{SOT}}(\mathcal{H})$ and either $X_{i}=\sum_{k=0}^{\infty} \sum_{\alpha \in \mathbb{F}_{n}^{+},|\alpha|=k} c_{\alpha}^{(i)}[\varphi(X)]_{\alpha}, i=1, \ldots, n$, where the convergence of the series is in the strong operator topology, or $\varphi(X) \in \mathcal{C}_{\psi}^{\text {rad }}(\mathcal{H})$ and

$$
X_{i}=\mathrm{SOT}-\lim _{r \rightarrow 1} \sum_{k=0}^{\infty} \sum_{\alpha \in \mathbb{F}_{n}^{+},|\alpha|=k} c_{\alpha}^{(i)} r^{|\alpha|}[\varphi(X)]_{\alpha}, \quad i=1, \ldots, n
$$

(b) $X \in \mathcal{C}_{\varphi}^{\mathrm{rad}}(\mathcal{H})$ and either $X_{i}=\sum_{k=0}^{\infty} \sum_{\alpha \in \mathbb{F}_{n}^{+}, \alpha \mid=k} c_{\alpha}^{(i)}[\widehat{\varphi}(X)]_{\alpha}, i=1, \ldots, n$, where the convergence of the series is in the strong operator topology, or $\widehat{\varphi}(X) \in \mathcal{C}_{\psi}^{\text {rad }}(\mathcal{H})$ and

$$
X_{i}=\mathrm{SOT}-\lim _{r \rightarrow 1} \sum_{k=0}^{\infty} \sum_{\alpha \in \mathbb{F}_{n}^{+},|\alpha|=k} c_{\alpha}^{(i)} r^{|\alpha|}[\widehat{\varphi}(X)]_{\alpha}, \quad i=1, \ldots, n
$$

Definition 2.3 (see [28]) Let $f:=\sum_{\alpha \in \mathbb{F}_{n}^{+}} a_{\alpha} Z_{\alpha}$ be a positive regular free holomorphic function, and let $\varphi=\left(\varphi_{1}, \ldots, \varphi_{n}\right)$ be an $n$-tuple of formal power series with model property. The noncommutative domain $\mathbb{D}_{f, \varphi}(\mathcal{H})$ is the set of all $n$-tuples of bounded linear operators $X=\left(X_{1}, \ldots, X_{n}\right) \in B(\mathcal{H})^{n}$ such that $\psi(\varphi(X))=X$ and

$$
\sum_{|\alpha| \geq 1} a_{\alpha}[\varphi(X)]_{\alpha}[\varphi(X)]_{\alpha}^{*} \leq I_{\mathcal{H}}
$$

where the convergence is in the weak operator topology. Define the strict noncommutative domain

$$
\mathbb{D}_{f, \varphi}^{<}(\mathcal{H}):=\left\{X \in B(\mathcal{H})^{n}: \psi(\varphi(X))=X \text { and }\left\|\sum_{|\alpha| \geq 1} a_{\alpha}[\varphi(X)]_{\alpha}[\varphi(X)]_{\alpha}^{*}\right\|<1\right\}
$$

where the convergence is in the weak operator topology. 
We define the noncommutative Hardy algebra $H^{\infty}\left(\mathbb{D}_{f, \varphi}\right)$ to be the WOT-closure of all noncommutative polynomials in $M_{Z_{1}}, \ldots, M_{Z_{n}}$ and the identity. Similarly, we can also define the noncommutative Hardy algebra $R^{\infty}\left(\mathbb{D}_{f, \varphi}\right)$ to be the WOT-closure of all noncommutative polynomials in $R_{Z_{1}}, \ldots, R_{Z_{n}}$ and the identity. Now we can define the strict noncommutative variety

$$
\mathcal{V}_{f, \varphi, \mathcal{I}}^{<}(\mathcal{H}):=\left\{\left(X_{1}, \ldots, X_{n}\right) \in \mathbb{D}_{f, \varphi}^{<}(\mathcal{H}): \omega\left(X_{1}, \ldots, X_{n}\right)=0 \text { for any } \omega \in \mathcal{I}\right\}
$$

where $\mathcal{I}$ is a WOT-closed two-sided ideal of the noncommutative Hardy algebra $H^{\infty}\left(\mathbb{D}_{f, \varphi}\right)$.

\subsection{Noncommutative Poisson kernel}

If $T=\left(T_{1}, \ldots, T_{n}\right) \in \mathbb{D}_{f, \varphi}(\mathcal{H})$, we define the positive linear mapping

$$
\Phi_{f, \varphi, T}: B(\mathcal{H}) \rightarrow B(\mathcal{H}) \quad \text { by } \Phi_{f, \varphi, T}(Y):=\sum_{|\alpha| \geq 1} a_{\alpha}[\varphi(T)]_{\alpha} Y[\varphi(T)]_{\alpha}^{*}
$$

where the convergence is in the weak operator topology. We say that $T=\left(T_{1}, \ldots, T_{n}\right)$ is a pure $n$-tuple of operators in $\mathbb{D}_{f, \varphi}(\mathcal{H})$ if

$$
\text { SOT- } \lim _{m \rightarrow \infty} \Phi_{f, \varphi, T}^{m}(I)=0 .
$$

The set of all pure elements of $\mathbb{D}_{f, \varphi}(\mathcal{H})$ is denoted by $\mathbb{D}_{f, \varphi}^{\text {pure }}(\mathcal{H})$. Notice that $\left(M_{Z_{1}}, \ldots, M_{Z_{n}}\right)$ is in $\mathbb{D}_{f, \varphi}^{\text {pure }}\left(\mathbb{H}_{f}^{2}(\varphi)\right)$. Moreover, we refer to the $n$-tuple $\left(M_{Z_{1}}, \ldots, M_{Z_{n}}\right)$ as the universal model associated with the abstract noncommutative domain $\mathbb{D}_{f, \varphi}$. An $n$-tuple $T \in \mathbb{D}_{f, \varphi}(\mathcal{H})$ is called completely non-coisometric (c.n.c.) if there is no vector $h \in \mathcal{H}, h \neq 0$, such that

$$
\left\langle\Phi_{f, \varphi, T}^{m}(I) h, h\right\rangle=\|h\|^{2} \quad \text { for any } m=1,2, \ldots
$$

The set of all c.n.c. elements of $\mathbb{D}_{f, \varphi}(\mathcal{H})$ is denoted by $\mathbb{D}_{f, \varphi}^{\mathrm{cnc}}(\mathcal{H})$. Note that

$$
\mathbb{D}_{f, \varphi}^{\text {pure }}(\mathcal{H}) \subseteq \mathbb{D}_{f, \varphi}^{\text {cnc }}(\mathcal{H}) \subseteq \mathbb{D}_{f, \varphi}(\mathcal{H})
$$

Similarly, we have

$$
\mathcal{V}_{f, \varphi, \mathcal{I}}^{\text {pure }}(\mathcal{H}) \subseteq \mathcal{V}_{f, \varphi, \mathcal{I}}^{\mathrm{cnc}}(\mathcal{H}) \subseteq \mathcal{V}_{f, \varphi, \mathcal{I}}(\mathcal{H})
$$

Moreover, it is obvious that the $n$-tuple $\left(B_{1}, \ldots, B_{n}\right)$ is in the noncommutative variety $\mathcal{V}_{f, \varphi, \mathcal{I}}^{\text {pure }}\left(\mathcal{N}_{f, \varphi, \mathcal{I}}\right)$, where $B_{i}:=\left.P_{\mathcal{N}_{f, \varphi, \mathcal{I}}} M_{Z_{i}}\right|_{\mathcal{N}_{f, \varphi, \mathcal{I}}}$ for $i=1, \ldots, n$. We refer to the $n$-tuple $\left(B_{1}, \ldots, B_{n}\right)$ as the universal model associated with the abstract noncommutative variety $\mathcal{V}_{f, \varphi, \mathcal{I} \text {. }}$

We define the noncommutative Poisson kernel associated with the $n$-tuple $T:=$ $\left(T_{1}, \ldots, T_{n}\right) \in \mathbb{D}_{f, \varphi}(\mathcal{H})$ to be the operator $K_{f, \varphi, T}: \mathcal{H} \rightarrow \mathbb{H}_{f}^{2}(\varphi) \otimes \overline{\Delta_{f, \varphi, T}(\mathcal{H})}$ defined by

$$
K_{f, \varphi, T} h:=\sum_{\alpha \in \mathbb{F}_{n}^{+}} b_{\alpha} \varphi_{\alpha} \otimes \Delta_{f, \varphi, T}[\varphi(T)]_{\alpha}^{*} h, \quad h \in \mathcal{H},
$$

where $\Delta_{f, \varphi, T}:=\left(I-\Phi_{f, \varphi, T}(I)\right)^{\frac{1}{2}}$ and the coefficients $b_{\alpha}, \alpha \in \mathbb{F}_{n}^{+}$, are given by relation (2.1). 


\subsection{Characteristic function}

We consider the full Fock space of $H_{n}$ defined by

$$
F^{2}\left(H_{n}\right):=\mathbb{C} 1 \oplus \bigoplus_{m \geq 1} H_{n}^{\otimes m}
$$

where $H_{n}^{\otimes m}$ is the Hilbert tensor product of $m$ copies of $H_{n}$. Define the left creation operators $S_{i}, i=1, \ldots, n$, acting on $F^{2}\left(H_{n}\right)$ by setting $S_{i} \xi:=e_{i} \otimes \xi, \xi \in F^{2}\left(H^{n}\right)$. If $A \in$ $B\left(F^{2}\left(H_{n}\right) \otimes \mathcal{G}, F^{2}\left(H_{n}\right) \otimes \mathcal{K}\right)$ and

$$
\left(S_{i}^{*} \otimes I_{\mathcal{K}}\right) A\left(S_{j} \otimes I_{\mathcal{G}}\right)=\delta_{i j} A, \quad i, j=1, \ldots, n,
$$

then $A$ is called multi-Toeplitz with respect to $S_{1}, \ldots, S_{n}$. Moreover, if $A \in B\left(F^{2}\left(H_{n}\right) \otimes \mathcal{G}\right.$, $\left.F^{2}\left(H_{n}\right) \otimes \mathcal{K}\right)$ and

$$
A\left(S_{i} \otimes I_{\mathcal{G}}\right)=\left(S_{i} \otimes I_{\mathcal{K}}\right) A, \quad i=1, \ldots, n,
$$

then $A$ is called multi-analytic with respect to $S_{1}, \ldots, S_{n}$ (see $\left.[17,19]\right)$. We remark that several results concerning the full Fock space $F^{2}\left(H_{n}\right)$ have been extended to the Hilbert space $\mathbb{H}_{f}^{2}(\varphi)$ (see $\left.[25,26,28]\right)$. If $A \in B\left(\mathbb{H}_{f}^{2}(\varphi) \otimes \mathcal{G}, \mathbb{H}_{f}^{2}(\varphi) \otimes \mathcal{K}\right)$, and

$$
A\left(M_{Z_{i}} \otimes I_{\mathcal{G}}\right)=\left(M_{Z_{i}} \otimes I_{\mathcal{K}}\right) A, \quad i=1, \ldots, n,
$$

then $A$ is called multi-analytic with respect to $M_{Z_{1}}, \ldots, M_{Z_{n}}$ (see Definition 3.1 of [28]). Indeed, this definition is an analogy.

Let $f=\sum_{|\alpha| \geq 1} a_{\alpha} X_{\alpha}$ be a positive regular free holomorphic function and define the set $\Gamma:=\left\{\alpha \in \mathbb{F}_{n}^{+}: a_{\alpha} \neq 0\right\}$ and $N:=\operatorname{card}(\Gamma)$. If $\varphi=\left(\varphi_{1}, \ldots, \varphi_{n}\right)$ is an $n$-tuple of formal power series with the model property and $T:=\left(T_{1}, \ldots, T_{n}\right) \in \mathbb{D}_{f, \varphi}(\mathcal{H})$, we define the row operator

$$
C_{f, \varphi, T}:=\left[\sqrt{a_{\tilde{\alpha}}}[\varphi(T)]_{\tilde{\alpha}}: \alpha \in \Gamma\right],
$$

where the entries are arranged in the lexicographic order of $\Gamma \subset \mathbb{F}_{n}^{+}$, and $\widetilde{\alpha}$ is the reverse of $\alpha=g_{i_{1}} \cdots g_{i_{k}}$, i.e., $\widetilde{\alpha}=g_{i_{k}} \cdots g_{i_{1}}$. Note that $C_{f, \varphi, T}$ is an operator acting from $\mathcal{H}^{(N)}$ (the completion of the direct sum of $N$ copies of $\mathcal{H}$ ) to $\mathcal{H}$.

Let $\left(M_{Z_{1}}, \ldots, M_{Z_{n}}\right)$ be the universal model associated with the abstract noncommutative domain $\mathbb{D}_{f, \varphi}$. We introduce the characteristic function of an $n$-tuple $T:=\left(T_{1}, \ldots, T_{n}\right) \in$ $\mathbb{D}_{f, \varphi}(\mathcal{H})$ to be the multi-analytic operator with respect to $M_{Z_{1}}, \ldots, M_{Z_{n}}$,

$$
\Theta_{f, \varphi, T}: \mathbb{H}_{f}^{2}(\varphi) \otimes \mathcal{D}_{C_{f, \varphi, T}^{*}} \rightarrow \mathbb{H}_{f}^{2}(\varphi) \otimes \mathcal{D}_{C_{f, \varphi, T}}
$$

with formal Fourier representation

$$
\begin{aligned}
-I & \otimes C_{f, \varphi, T}+\left(I \otimes \Delta_{C_{f, \varphi, T}}\right)\left(I-\sum_{|\alpha| \geq 1} a_{\widetilde{\alpha}} R_{\varphi_{\alpha}} \otimes[\varphi(T)]_{\tilde{\alpha}}^{*}\right)^{-1} \\
& \times\left[\sqrt{a_{\widetilde{\alpha}}} R_{\varphi_{\alpha}} \otimes I: \alpha \in \Gamma\right]\left(I \otimes \Delta_{C_{f, \varphi, T}^{*}}\right),
\end{aligned}
$$


where $R_{\varphi_{1}}, \ldots, R_{\varphi_{n}}$ are the right multiplication operators by the formal power series $\varphi_{1}, \ldots, \varphi_{n}$, respectively, on the Hilbert space $\mathbb{H}_{f}^{2}(\varphi)$. The defect operators associated with the row contraction $C_{f, \varphi, T}$ are

$$
\begin{aligned}
\Delta_{C_{f, \varphi, T}} & :=\left(I-C_{f, \varphi, T} C_{f, \varphi, T}^{*}\right)^{\frac{1}{2}} \in B(\mathcal{H}), \\
\Delta_{C_{f, \varphi, T}^{*}} & :=\left(I-C_{f, \varphi, T}^{*} C_{f, \varphi, T}\right)^{\frac{1}{2}} \in B\left(\mathcal{H}^{(N)}\right),
\end{aligned}
$$

and the defect spaces are $\mathcal{D}_{C_{f, \varphi, T}}:=\overline{\Delta_{C_{f, \varphi, T}} \mathcal{H}}$ and $\mathcal{D}_{C_{f, \varphi, T}^{*}}:=\overline{\Delta_{C_{f, \varphi, T}^{*}} \mathcal{H}^{(N)}}$.

\section{Constrained characteristic functions}

In this section, we present a functional model theorem for completely non-coisometric $n$ tuples of operators in the noncommutative variety $\mathcal{V}_{f, \varphi, \mathcal{I}}(\mathcal{H})$ in terms of constrained characteristic functions. Moreover, we prove that the constrained characteristic function is a complete unitary invariant for this class of elements. Indeed, this result can be viewed as the noncommutative analogue of the classical Sz.-Nagy-Foiaş functional model for completely nonunitary contractions.

Let $T=\left(T_{1}, \ldots, T_{n}\right)$ be an $n$-tuple of operators in $\mathcal{V}_{f, \varphi, \mathcal{I}}^{\mathrm{cnc}}(\mathcal{H})$. The constrained Poisson kernel is the operator $K_{f, \varphi, T}^{(\mathcal{I})}: \mathcal{H} \rightarrow \mathcal{N}_{f, \varphi, \mathcal{I}} \otimes \mathcal{D}_{C_{f, \varphi, T}}$ defined by

$$
K_{f, \varphi, T}^{(\mathcal{I})}:=\left(P_{\mathcal{N}_{f, \varphi, \mathcal{I}}} \otimes I_{\mathcal{D}_{C f, \varphi, T}}\right) K_{f, \varphi, T}
$$

where $K_{f, \varphi, T}$ is the noncommutative Poisson kernel associated with $f, \varphi$, and $T$.

First, we present some basic properties for the constrained Poisson kernel $K_{f, \varphi, T}^{(\mathcal{I})}$ associated with $f, \varphi, T$, and $\mathcal{I}$.

Theorem 3.1 Letf $:=\sum_{\alpha \in \mathbb{F}_{n}^{+}} a_{\alpha} Z_{\alpha}$ be a positive regular free holomorphic function, and let $\varphi=\left(\varphi_{1}, \ldots, \varphi_{n}\right)$ be an $n$-tuple offormal power series with model property. Let $\mathcal{I} \neq H^{\infty}\left(\mathbb{D}_{f, \varphi}\right)$ be a WOT-closed two-sided ideal of the noncommutative Hardy algebra $H^{\infty}\left(\mathbb{D}_{f, \varphi}\right)$. If $T=$ $\left(T_{1}, \ldots, T_{n}\right)$ is an $n$-tuple of operators in $\mathcal{V}_{f, \varphi, \mathcal{I}}^{\mathrm{cnc}}(\mathcal{H})$, then the following statements hold:

(i) $K_{f, \varphi, T}^{(\mathcal{I})} T_{i}^{*}=\left(B_{i}^{*} \otimes I_{\mathcal{D}_{C f, \varphi, T}}\right) K_{f, \varphi, T}^{(\mathcal{I})}, i=1, \ldots, n$;

(ii) $K_{f, \varphi, T}^{(\mathcal{I})}$ is an isometry if and only if $T$ is pure, where $K_{f, \varphi, T}^{(\mathcal{I})}$ is the constrained Poisson kernel associated with $f, \varphi, T$, and $\mathcal{I}$.

Proof (i) According to the proof of Theorem 2.1 from [28], we know that

$$
K_{f, \varphi, T} T_{i}^{*}=\left(M_{Z_{i}}^{*} \otimes I_{\mathcal{D}_{f, \varphi, T}}\right) K_{f, \varphi, T}, \quad i=1, \ldots, n,
$$

where $K_{f, \varphi, T}$ is the noncommutative Poisson kernel associated with $f, \varphi$, and $T$. Hence, we have

$$
K_{f, \varphi, T}^{*}\left(p\left(M_{Z_{1}}, \ldots, M_{Z_{n}}\right) \otimes I_{\mathcal{D}_{C_{f, \varphi, T}}}\right)=p\left(T_{1}, \ldots, T_{n}\right) K_{f, \varphi, T}^{*}
$$

for any polynomial $p$ in $M_{Z_{1}}, \ldots, M_{Z_{n}}$. Assume that

$$
\phi\left(V_{1}, \ldots, V_{n}\right)=\sum_{k=0}^{\infty} \sum_{|\alpha|=k} d_{\alpha} V_{\alpha}, \quad d_{\alpha} \in \mathbb{C},
$$


is an element in the noncommutative Hardy algebra $F^{\infty}\left(\mathcal{D}_{f}\right)$. Then we deduce that

$$
\phi\left(r V_{1}, \ldots, r V_{n}\right)=\sum_{k=0}^{\infty} \sum_{|\alpha|=k} r^{|\alpha|} d_{\alpha} V_{\alpha} \quad \text { for any } 0<r<1
$$

is in the noncommutative domain algebra $\mathcal{A}\left(\mathcal{D}_{f}\right)$. Moreover, since $\varphi$ has model property, we have

$$
M_{\varphi_{i}}=\varphi_{i}\left(M_{Z_{1}}, \ldots, M_{Z_{n}}\right), \quad i=1, \ldots, n,
$$

where $\left(M_{Z_{1}}, \ldots, M_{Z_{n}}\right)$ is either in the $\operatorname{set} \mathcal{C}_{\varphi}^{\mathrm{SOT}}\left(\mathbb{H}_{f}^{2}(\varphi)\right)$ or $\mathcal{C}_{\varphi}^{\mathrm{rad}}\left(\mathbb{H}_{f}^{2}(\varphi)\right)$. Using (2.3), we conclude that

$$
V_{i}=U \varphi_{i}\left(M_{Z_{1}}, \ldots, M_{Z_{n}}\right) U^{-1}, \quad i=1, \ldots, n
$$

Therefore, we obtain

$$
\phi\left(r \varphi_{1}\left(M_{Z}\right), \ldots, r \varphi_{n}\left(M_{Z}\right)\right)=\sum_{k=0}^{\infty} \sum_{|\alpha|=k} r^{|\alpha|} d_{\alpha}\left[\varphi\left(M_{Z}\right)\right]_{\alpha^{\prime}}
$$

where the series is convergent in the operator norm topology. Hence, due to (3.1), we infer that

$$
K_{f, \varphi, T}^{*}\left[\phi\left(r \varphi_{1}\left(M_{Z}\right), \ldots, r \varphi_{n}\left(M_{Z}\right)\right) \otimes I_{\mathcal{D}_{C_{f, \varphi}, T}}\right]=\phi\left(r \varphi_{1}(T), \ldots, r \varphi_{n}(T)\right) K_{f, \varphi, T}^{*}
$$

for any $\phi\left(V_{1}, \ldots, V_{n}\right) \in F^{\infty}\left(\mathcal{D}_{f}\right)$ and $0<r<1$. Since $T=\left(T_{1}, \ldots, T_{n}\right)$ is in $\mathbb{D}_{f, \varphi}^{\text {cnc }}(\mathcal{H})$ and $M_{Z}=$ $\left(M_{Z_{1}}, \ldots, M_{Z_{n}}\right)$ is in $\mathbb{D}_{f, \varphi}^{\text {pure }}\left(\mathbb{H}_{f}^{2}(\varphi)\right)$, we deduce that $\varphi(T)=\left(\varphi_{1}(T), \ldots, \varphi_{n}(T)\right)$ is a completely non-coisometric $n$-tuple of operators in the noncommutative domain $\mathcal{D}_{f}(\mathcal{H})$ and $\varphi\left(M_{Z}\right)=$ $\left(\varphi_{1}\left(M_{Z}\right), \ldots, \varphi_{n}\left(M_{Z}\right)\right)$ is a pure $n$-tuple of operators in $\mathcal{D}_{f}\left(\mathbb{H}_{f}^{2}(\varphi)\right)$. Taking into account that

$$
\left\|\phi\left(r \varphi_{1}\left(M_{Z}\right), \ldots, r \varphi_{n}\left(M_{Z}\right)\right)\right\| \leq\left\|\phi\left(V_{1}, \ldots, V_{n}\right)\right\|
$$

and using $F^{\infty}\left(\mathcal{D}_{f}\right)$-functional calculus (see [24]), we infer that

$$
K_{f, \varphi, T}^{*}\left[\phi\left(\varphi_{1}\left(M_{Z}\right), \ldots, \varphi_{n}\left(M_{Z}\right)\right) \otimes I_{\mathcal{D}_{C_{f, \varphi, T}}}\right]=\phi\left(\varphi_{1}(T), \ldots, \varphi_{n}(T)\right) K_{f, \varphi, T}^{*}
$$

for any $\phi\left(V_{1}, \ldots, V_{n}\right) \in F^{\infty}\left(\mathcal{D}_{f}\right)$. Using Proposition 4.2 from [28], we know that if $\theta \in$ $H^{\infty}\left(\mathbb{D}_{f, \varphi}\right)$, there is $\chi=\sum_{\alpha \in \mathbb{F}_{n}^{+}} c_{\alpha} V_{\alpha}$ in $F^{\infty}\left(\mathcal{D}_{f}\right)$ such that

$$
\theta=\mathrm{SOT}-\lim _{r \rightarrow 1} \sum_{k=0}^{\infty} \sum_{|\alpha|=k} c_{\alpha} r^{|\alpha|}\left[\varphi\left(M_{Z}\right)\right]_{\alpha}=\chi\left(\varphi\left(M_{Z}\right)\right)
$$

Indeed, this implies that

$$
H^{\infty}\left(\mathbb{D}_{f, \varphi}\right)=\left\{\chi\left(\varphi\left(M_{Z}\right)\right): \chi \in F^{\infty}\left(\mathcal{D}_{f}\right)\right\} .
$$


Moreover, since $T=\left(T_{1}, \ldots, T_{n}\right)$ is in $\mathcal{V}_{f, \varphi, \mathcal{I}}^{\mathrm{cnc}}(\mathcal{H})$, we deduce that $\varphi(T)=\left(\varphi_{1}(T), \ldots, \varphi_{n}(T)\right)$ is also a completely non-coisometric $n$-tuple of operators in $\mathcal{D}_{f}(\mathcal{H})$. Using $F^{\infty}\left(\mathcal{D}_{f}\right)$ functional calculus, we obtain that

$$
\theta\left(T_{1}, \ldots, T_{n}\right)=\text { SOT }-\lim _{r \rightarrow 1} \sum_{k=0}^{\infty} \sum_{|\alpha|=k} c_{\alpha} r^{|\alpha|}[\varphi(T)]_{\alpha}=\chi\left(\varphi_{1}(T), \ldots, \varphi_{n}(T)\right)
$$

This shows that

$$
K_{f, \varphi, T}^{*}\left(\omega \otimes I_{\mathcal{D}_{C_{f, \varphi, T}}}\right)=\omega(T) K_{f, \varphi, T}^{*}
$$

for any $\omega \in H^{\infty}\left(\mathbb{D}_{f, \varphi}\right)$. Consequently, we deduce that

$$
\left\langle\left(\omega^{*} \otimes I_{\mathcal{D}_{C_{f, \varphi, T}}}\right) K_{f, \varphi, T} h, 1 \otimes d\right\rangle=\left\langle K_{f, \varphi, T} \omega(T)^{*} h, 1 \otimes d\right\rangle
$$

for any $\omega \in H^{\infty}\left(\mathbb{D}_{f, \varphi}\right), h \in \mathcal{H}$, and $d \in \mathcal{D}_{C_{f, \varphi, T}}$. Since $\mathcal{I}$ is a WOT-closed two-sided ideal of $H^{\infty}\left(\mathbb{D}_{f, \varphi}\right)$, we have

$$
\mathcal{M}_{f, \varphi, \mathcal{I}}=\overline{\mathcal{I}(1)}
$$

Note that $T \in \mathcal{V}_{f, \varphi, \mathcal{I}}^{\mathrm{cnc}}(\mathcal{H})$. Then we obtain

$$
\left\langle K_{f, \varphi, T} h, \omega(1) \otimes d\right\rangle=0
$$

for any $\omega \in \mathcal{I}, h \in \mathcal{H}$, and $d \in \mathcal{D}_{C_{f, \varphi, T}}$. Therefore, we conclude that

$$
K_{f, \varphi, T}(\mathcal{H}) \subseteq \mathcal{N}_{f, \varphi, \mathcal{I}} \otimes \mathcal{D}_{C_{f, \varphi, T}}
$$

which implies that

$$
K_{f, \varphi, T}^{(\mathcal{I})} h=\left(P_{\mathcal{N}_{f, \varphi, \mathcal{I}}} \otimes I_{\mathcal{D}_{C_{f, \varphi, T}}}\right) K_{f, \varphi, T} h=K_{f, \varphi, T} h, \quad h \in \mathcal{H}
$$

On the other hand, since $\mathcal{N}_{f, \varphi, \mathcal{I}}$ is an invariant subspace under $M_{Z_{1}}^{*}, \ldots, M_{Z_{n}}^{*}$, we have

$$
B_{\alpha}=\left.P_{\mathcal{N}_{f, \varphi, \mathcal{I}}} M_{Z_{\alpha}}\right|_{\mathcal{N}_{f, \varphi, \mathcal{I}}} \quad \text { for any } \alpha \in \mathbb{F}_{n}^{+}
$$

According to Proposition 4.2 of [28], we know that, for any $v \in H^{\infty}\left(\mathbb{D}_{f, \varphi}\right)$, there exists $\chi \in F^{\infty}\left(\mathcal{D}_{f}\right)$ such that

$$
\begin{aligned}
v\left(M_{Z_{1}}, \ldots, M_{Z_{n}}\right) & =\chi\left(\varphi_{1}\left(M_{Z}\right), \ldots, \varphi_{n}\left(M_{Z}\right)\right) \\
& =\text { SOT }-\lim _{r \rightarrow 1} \chi\left(r \varphi_{1}\left(M_{Z}\right), \ldots, r \varphi_{n}\left(M_{Z}\right)\right) .
\end{aligned}
$$

Since $\left(B_{1}, \ldots, B_{n}\right)$ is in the noncommutative variety $\mathcal{V}_{f, \varphi, \mathcal{I}}^{\text {pure }}\left(\mathcal{N}_{f, \varphi, \mathcal{I}}\right)$, we obtain that $\left(\varphi_{1}(B), \ldots, \varphi_{n}(B)\right)$ is a pure $n$-tuple of operators in $\mathcal{D}_{f}\left(\mathcal{N}_{f, \varphi, \mathcal{I}}\right)$. Consequently, using $F^{\infty}\left(\mathcal{D}_{f}\right)$ functional calculus, we deduce that

$$
\nu\left(B_{1}, \ldots, B_{n}\right)=\left.P_{\mathcal{N}_{f, \varphi, \mathcal{I}}} v\left(M_{Z_{1}}, \ldots, M_{Z_{n}}\right)\right|_{\mathcal{N}_{f, \varphi, \mathcal{I}}}
$$


for any $v \in H^{\infty}\left(\mathbb{D}_{f, \varphi}\right)$. Applying (3.2), (3.3), and (3.4), we infer that

$$
\begin{aligned}
K_{f, \varphi, T}^{(\mathcal{I})} v\left(T_{1}, \ldots, T_{n}\right)^{*}= & \left(P_{\mathcal{N}_{f, \varphi, \mathcal{I}}} \otimes I_{\mathcal{D}_{C_{f, \varphi}, T}}\right)\left[v\left(M_{Z_{1}}, \ldots, M_{Z_{n}}\right)^{*} \otimes I_{\mathcal{D}_{C_{f, \varphi}, T}}\right] \\
& \times\left(P_{\mathcal{N}_{f, \varphi, \mathcal{I}}} \otimes I_{\mathcal{D}_{C_{f, \varphi}, T}}\right) K_{f, \varphi, T} \\
= & {\left[v\left(B_{1}, \ldots, B_{n}\right)^{*} \otimes I_{\mathcal{D}_{C_{f, \varphi}, T}}\right] K_{f, \varphi, T}^{(\mathcal{I})} }
\end{aligned}
$$

for any $v\left(B_{1}, \ldots, B_{n}\right) \in H^{\infty}\left(\mathcal{V}_{f, \varphi, \mathcal{I}}\right)$. In particular, we have

$$
K_{f, \varphi, T}^{(\mathcal{I})} T_{i}^{*}=\left(B_{i}^{*} \otimes I_{\mathcal{D}_{C f, \varphi}}\right) K_{f, \varphi, T}^{(\mathcal{I})}, \quad i=1, \ldots, n
$$

(ii) Due to (3.3), we obtain

$$
\begin{aligned}
\left\langle\left(K_{f, \varphi, T}^{(\mathcal{I})}\right)^{*} K_{f, \varphi, T}^{(\mathcal{I})} h, h\right\rangle & =\left\|K_{f, \varphi, T} h\right\|^{2} \\
& =\|h\|^{2}-\lim _{m \rightarrow \infty}\left\langle\Phi_{f, \varphi, T}^{m}(I) h, h\right\rangle .
\end{aligned}
$$

Hence, we deduce that

$$
\left(K_{f, \varphi, T}^{(\mathcal{I})}\right)^{*} K_{f, \varphi, T}^{(\mathcal{I})}=I-\Phi_{f, \varphi, T}^{\infty}(I)
$$

where $\Phi_{f, \varphi, T}^{\infty}(I):=$ SOT- $\lim _{m \rightarrow \infty} \Phi_{f, \varphi, T}^{m}(I)$. Therefore, (ii) holds. This completes the proof.

We define the constrained characteristic function associated with an $n$-tuple $T:=$ $\left(T_{1}, \ldots, T_{n}\right) \in \mathcal{V}_{f, \varphi, \mathcal{I}}^{\mathrm{cnc}}(\mathcal{H})$ to be the multi-analytic operator with respect to the constrained weighted shifts $B_{1}, \ldots, B_{n}$,

$$
\Theta_{f, \varphi, T}^{(\mathcal{I})}: \mathcal{N}_{f, \varphi, \mathcal{I}} \otimes \mathcal{D}_{C_{f, \varphi, T}^{*}} \rightarrow \mathcal{N}_{f, \varphi, \mathcal{I}} \otimes \mathcal{D}_{C_{f, \varphi, T}}
$$

with the formal Fourier representation

$$
\begin{aligned}
& -I_{\mathcal{N}_{f, \varphi, \mathcal{I}}} \otimes C_{f, \varphi, T}+\left(I_{\mathcal{N}_{f, \varphi, \mathcal{I}}} \otimes \Delta_{C_{f, \varphi, T}}\right)\left(I_{\mathcal{N}_{f, \varphi, \mathcal{I}} \otimes \mathcal{H}}-\sum_{|\alpha| \geq 1} a_{\widetilde{\alpha}} D_{\alpha} \otimes[\varphi(T)]_{\tilde{\alpha}}^{*}\right)^{-1} \\
& \quad \times\left[\sqrt{a_{\widetilde{\alpha}}} D_{\alpha} \otimes I_{\mathcal{H}}: \alpha \in \Gamma\right]\left(I_{\mathcal{N}_{f, \varphi, \mathcal{I}}} \otimes \Delta_{C_{f, \varphi, T}^{*}}\right),
\end{aligned}
$$

where $D_{i}=\left.P_{\mathcal{N}_{f, \varphi, \mathcal{I}}} R_{\varphi_{i}}\right|_{\mathcal{N}_{f, \varphi, \mathcal{I}}}, i=1, \ldots, n$, and $R_{\varphi_{1}}, \ldots, R_{\varphi_{n}}$ are the right multiplication operators by the power series $\varphi_{1}, \ldots, \varphi_{n}$, respectively, on the Hilbert space $\mathbb{H}_{f}^{2}(\varphi)$.

We provide a factorization result for the constrained characteristic function, which will play an important role in our investigation.

Theorem 3.2 Let $f:=\sum_{\alpha \in \mathbb{F}_{n}^{+}} a_{\alpha} Z_{\alpha}$ be a positive regular free holomorphic function, and let $\varphi=\left(\varphi_{1}, \ldots, \varphi_{n}\right)$ be an $n$-tuple offormal power series with model property. Let $\mathcal{I} \neq H^{\infty}\left(\mathbb{D}_{f, \varphi}\right)$ be a WOT-closed two-sided ideal of the noncommutative Hardy algebra $H^{\infty}\left(\mathbb{D}_{f, \varphi}\right)$. Then

$$
I_{\mathcal{N}_{f, \varphi, \mathcal{I}} \otimes \mathcal{D}_{C f, \varphi, T}}-\Theta_{f, \varphi, T}^{(\mathcal{I})}\left(\Theta_{f, \varphi, T}^{(\mathcal{I})}\right)^{*}=K_{f, \varphi, T}^{(\mathcal{I})}\left(K_{f, \varphi, T}^{(\mathcal{I})}\right)^{*}
$$


where $\Theta_{f, \varphi, T}^{(\mathcal{I})}$ is the constrained characteristic function and $K_{f, \varphi, T}^{(\mathcal{I})}$ is the corresponding constrained Poisson kernel.

Proof Due to Theorem 6.1 of [28], we know that

$$
I_{\mathbb{H}_{f}^{2}(\varphi) \otimes \mathcal{D}_{C_{f, \varphi, T}}}-\Theta_{f, \varphi, T} \Theta_{f, \varphi, T}^{*}=K_{f, \varphi, T} K_{f, \varphi, T}^{*}
$$

According to the proof of Theorem 3.1, we have

$$
K_{f, \varphi, T}(\mathcal{H}) \subseteq \mathcal{N}_{f, \varphi, \mathcal{I}} \otimes \mathcal{D}_{C_{f, \varphi, T}} \subseteq \mathbb{H}_{f}^{2}(\varphi) \otimes \mathcal{D}_{C_{f, \varphi, T}}
$$

Hence, we infer that

$$
\begin{aligned}
& I_{\mathcal{N}_{f, \varphi, \mathcal{I}} \otimes \mathcal{D}_{C_{f, \varphi}, T}}-\left.P_{\mathcal{N}_{f, \varphi, \mathcal{I}} \otimes \mathcal{D}_{C_{f, \varphi, T}}} \Theta_{f, \varphi, T} \Theta_{f, \varphi, T}^{*}\right|_{\mathcal{N}_{f, \varphi, \mathcal{I}} \otimes \mathcal{D}_{C_{f, \varphi}, T}} \\
& =\left.P_{\mathcal{N}_{f, \varphi, \mathcal{I}} \otimes \mathcal{D}_{C_{f, \varphi}, T}} K_{f, \varphi, T} K_{f, \varphi, T}^{*}\right|_{\mathcal{N}_{f, \varphi, \mathcal{I}} \otimes \mathcal{D}_{C_{f, \varphi, T}}} .
\end{aligned}
$$

Since $\mathcal{N}_{f, \varphi, \mathcal{I}}$ is an invariant subspace under $R_{\varphi_{1}}^{*}, \ldots, R_{\varphi_{n}}^{*}$, we obtain

$$
\Theta_{f, \varphi, T}^{*}\left(\mathcal{N}_{f, \varphi, \mathcal{I}} \otimes \mathcal{D}_{C_{f, \varphi, T}}\right) \subseteq \mathcal{N}_{f, \varphi, \mathcal{I}} \otimes \mathcal{D}_{C_{f, \varphi, T}^{*}}
$$

and

$$
\left.P_{\mathcal{N}_{f, \varphi, \mathcal{I}} \otimes \mathcal{D}_{C_{f, \varphi, T}}} \Theta_{f, \varphi, T}\right|_{\mathcal{N}_{f, \varphi, \mathcal{I}} \otimes \mathcal{D}_{C_{f, \varphi, T}^{*}}}=\Theta_{f, \varphi, T}^{(\mathcal{I})}
$$

Applying (3.6), (3.7), and (3.8), we deduce that

$$
I_{\mathcal{N}_{f, \varphi, \mathcal{I}} \otimes \mathcal{D}_{C f, \varphi, T}}-\Theta_{f, \varphi, T}^{(\mathcal{I})}\left(\Theta_{f, \varphi, T}^{(\mathcal{I})}\right)^{*}=K_{f, \varphi, T}^{(\mathcal{I})}\left(K_{f, \varphi, T}^{(\mathcal{I})}\right)^{*} .
$$

This completes the proof.

If $A \in B\left(\mathbb{H}_{f}^{2}(\varphi) \otimes \mathcal{G}, \mathbb{H}_{f}^{2}(\varphi) \otimes \mathcal{K}\right)$ is a multi-analytic operator and $A$ is a partial isometry, then we call it inner multi-analytic.

In what follows, we present a functional model theorem for completely non-coisometric $n$-tuples of operators in the noncommutative variety $\mathcal{V}_{f, \varphi, \mathcal{I}}(\mathcal{H})$ in terms of constrained characteristic functions.

Theorem 3.3 Letf $:=\sum_{\alpha \in \mathbb{F}_{n}^{+}} a_{\alpha} Z_{\alpha}$ be a positive regular free holomorphic function, and let $\varphi=\left(\varphi_{1}, \ldots, \varphi_{n}\right)$ be an $n$-tuple of formal power series with model property. Let $\mathcal{I} \neq H^{\infty}\left(\mathbb{D}_{f, \varphi}\right)$ be a WOT-closed two-sided ideal of the noncommutative Hardy algebra $H^{\infty}\left(\mathbb{D}_{f, \varphi}\right)$. If $T:=$ $\left(T_{1}, \ldots, T_{n}\right)$ is in the noncommutative variety $\mathcal{V}_{f, \varphi, \mathcal{I}}^{\mathrm{cnc}}(\mathcal{H})$, then the following statements hold:

(i) $T$ is unitarily equivalent to the $n$-tuple $\widetilde{T}:=\left(\widetilde{T}_{1}, \ldots, \widetilde{T}_{n}\right) \in \mathcal{V}_{f, \varphi, \mathcal{I}}^{\mathrm{cnc}}(\widetilde{\mathcal{H}})$ on the Hilbert space

$$
\begin{aligned}
\widetilde{\mathcal{H}}:= & {\left[\left(\mathcal{N}_{f, \varphi, \mathcal{I}} \otimes \mathcal{D}_{\left.C_{f, \varphi, T}\right)} \oplus \overline{\Delta_{\Theta_{f, \varphi}^{(\mathcal{I}) T}}\left(\mathcal{N}_{f, \varphi, \mathcal{I}} \otimes \mathcal{D}_{C_{f, \varphi, T}^{*}}\right)}\right]\right.} \\
& \ominus\left\{\Theta_{f, \varphi, T}^{(\mathcal{I})} x \oplus \Delta_{\Theta_{f, \varphi, T}^{(\mathcal{I})}} x: x \in \mathcal{N}_{f, \varphi, \mathcal{I}} \otimes \mathcal{D}_{C_{f, \varphi, T}^{*}}\right\},
\end{aligned}
$$


where $\Delta_{\Theta_{f, \varphi, T}^{(\mathcal{I})}}=\left(I-\left(\Theta_{f, \varphi, T}^{(\mathcal{I})}\right)^{*} \Theta_{f, \varphi, T}^{(\mathcal{I})}\right)^{\frac{1}{2}}$ and each operator $\widetilde{T}_{i}, i=1, \ldots, n$, is uniquely defined by the relation

$$
\begin{aligned}
& \left(P_{\mathcal{N}_{f, \varphi, \mathcal{I}} \otimes \mathcal{D}_{C_{f, \varphi, T}} \mid \widetilde{\mathcal{H}}}\right) \widetilde{T}_{i}^{*} z \\
& =\left(B_{i}^{*} \otimes I_{\mathcal{D}_{C f, \varphi, T}}\right)\left(P_{\mathcal{N}_{f, \varphi}, \mathcal{I} \otimes \mathcal{D}_{C_{f, \varphi}, T} \mid \widetilde{\mathcal{H}}}\right) z, \quad z \in \widetilde{\mathcal{H}},
\end{aligned}
$$

where $\left.P_{\mathcal{N}_{f, \varphi, \mathcal{I}} \otimes \mathcal{D}_{C_{f, \varphi, T}}}\right|_{\tilde{\mathcal{H}}}$ is an injective operator, $P_{\mathcal{N}_{f, \varphi, \mathcal{I}} \otimes \mathcal{D}_{C_{f, \varphi}, T}}$ is the orthogonal projection from the Hilbert space

$$
\widetilde{\mathcal{K}}:=\left(\mathcal{N}_{f, \varphi, \mathcal{I}} \otimes \mathcal{D}_{C_{f, \varphi, T}}\right) \oplus \overline{\Delta_{\Theta_{f, \varphi, T}^{(\mathcal{I})}}\left(\mathcal{N}_{f, \varphi, \mathcal{I}} \otimes \mathcal{D}_{C_{f, \varphi, T}^{*}}\right)}
$$

onto the subspace $\mathcal{N}_{f, \varphi, \mathcal{I}} \otimes \mathcal{D}_{C_{f, \varphi, T}}$, and $B_{i}=\left.P_{\mathcal{N}_{f, \varphi, \mathcal{I}}} M_{Z_{i}}\right|_{\mathcal{N}_{f, \varphi}, \mathcal{I}}$ for any $i=1, \ldots, n$;

(ii) $T$ is in the noncommutative variety $\mathcal{V}_{f, \varphi, \mathcal{I}}^{\text {pure }}(\mathcal{H})$ if and only if the constrained characteristic function $\Theta_{f, \varphi, T}^{(\mathcal{I})}$ is an inner multi-analytic operator. In this case, $T$ is unitarily equivalent to the $n$-tuple

$$
\left(\left.P_{\widetilde{\mathcal{H}}}\left(B_{1} \otimes I_{\mathcal{D}_{C_{f, \varphi}, T}}\right)\right|_{\widetilde{\mathcal{H}}}, \ldots,\left.P_{\widetilde{\mathcal{H}}}\left(B_{n} \otimes I_{\mathcal{D}_{C_{f, \varphi}, T}}\right)\right|_{\widetilde{\mathcal{H}}}\right),
$$

where $P_{\widetilde{\mathcal{H}}}$ is the orthogonal projection from $\mathcal{N}_{f, \varphi, \mathcal{I}} \otimes \mathcal{D}_{C_{f, \varphi, T}}$ onto the Hilbert space $\widetilde{\mathcal{H}}:=\left(\mathcal{N}_{f, \varphi, \mathcal{I}} \otimes \mathcal{D}_{C_{f, \varphi, T}}\right) \ominus \Theta_{f, \varphi, T}^{(\mathcal{I})}\left(\mathcal{N}_{f, \varphi, \mathcal{I}} \otimes \mathcal{D}_{C_{f, \varphi}^{*}, T}\right)$.

Proof (i) We define the operator $\Psi: \mathcal{N}_{f, \varphi, \mathcal{I}} \otimes \mathcal{D}_{C_{f, \varphi, T}^{*}} \rightarrow \widetilde{\mathcal{K}}$ by setting

$$
\Psi x:=\Theta_{f, \varphi, T}^{(\mathcal{I})} x \oplus \Delta_{\Theta_{f, \varphi, T}^{(\mathcal{I})}} x, \quad x \in \mathcal{N}_{f, \varphi, \mathcal{I}} \otimes \mathcal{D}_{C_{f, \varphi, T}^{*}} .
$$

It is obvious that $\Psi$ is an isometry and

$$
\Psi^{*}(y \oplus 0)=\left(\Theta_{f, \varphi, T}^{(\mathcal{I})}\right)^{*} y, \quad y \in \mathcal{N}_{f, \varphi, \mathcal{I}} \otimes \mathcal{D}_{C_{f, \varphi, T}}
$$

Hence, we infer that

$$
\begin{aligned}
\|y\|^{2} & =\left\|P_{\widetilde{\mathcal{H}}}(y \oplus 0)\right\|^{2}+\left\|\Psi \Psi^{*}(y \oplus 0)\right\|^{2} \\
& =\left\|P_{\widetilde{\mathcal{H}}}(y \oplus 0)\right\|^{2}+\left\|\left(\Theta_{f, \varphi, T}^{(\mathcal{I})}\right)^{*} y\right\|^{2}
\end{aligned}
$$

for any $y \in \mathcal{N}_{f, \varphi, \mathcal{I}} \otimes \mathcal{D}_{C_{f, \varphi}, T}$, where $P_{\widetilde{\mathcal{H}}}$ denotes the orthogonal projection from $\widetilde{\mathcal{K}}$ onto $\widetilde{\mathcal{H}}$. According to Theorem 3.2, we have

$$
\left\|\left(K_{f, \varphi, T}^{(\mathcal{I})}\right)^{*} y\right\|^{2}+\left\|\left(\Theta_{f, \varphi, T}^{(\mathcal{I})}\right)^{*} y\right\|^{2}=\|y\|^{2}, \quad y \in \mathcal{N}_{f, \varphi, \mathcal{I}} \otimes \mathcal{D}_{C_{f, \varphi, T}} .
$$

Therefore, using (3.10) and (3.11), we deduce that

$$
\left\|\left(K_{f, \varphi, T}^{(\mathcal{I})}\right)^{*} y\right\|=\left\|P_{\widetilde{\mathcal{H}}}(y \oplus 0)\right\|, \quad y \in \mathcal{N}_{f, \varphi, \mathcal{I}} \otimes \mathcal{D}_{C_{f, \varphi}, T} .
$$

On the other hand, due to (3.3), we obtain

$$
\left\|K_{f, \varphi, T}^{(\mathcal{I})} h\right\|^{2}=\|h\|^{2}-\lim _{m \rightarrow \infty}\left\langle\Phi_{f, \varphi, T}^{m}(I) h, h\right\rangle, \quad h \in \mathcal{H} .
$$


Hence, if $K_{f, \varphi, T}^{(\mathcal{I})} h=0$, then we have

$$
\|h\|^{2}=\lim _{m \rightarrow \infty}\left\langle\Phi_{f, \varphi, T}^{m}(I) h, h\right\rangle .
$$

Since $T$ is in $\mathcal{V}_{f, \varphi, \mathcal{I}}^{\mathrm{cnc}}(\mathcal{H})$, we infer that $h=0$, which implies that $K_{f, \varphi, T}^{(\mathcal{I})}$ is an injective operator and range $\left(K_{f, \varphi, T}^{(\mathcal{I})}\right)^{*}$ is dense in $\mathcal{H}$.

Let $z \in \widetilde{\mathcal{H}}$ and assume that $z \perp P_{\tilde{\mathcal{H}}}(y \oplus 0)$ for any $y \in \mathcal{N}_{f, \varphi, \mathcal{I}} \otimes \mathcal{D}_{C_{f, \varphi, T}}$. Taking into account that

$$
\widetilde{\mathcal{K}}=\left\{y \oplus 0: y \in \mathcal{N}_{f, \varphi, \mathcal{I}} \otimes \mathcal{D}_{C_{f, \varphi, T}}\right\} \vee\left\{\Theta_{f, \varphi, T}^{(\mathcal{I})} x \oplus \Delta_{\Theta_{f, \varphi, T}^{(\mathcal{I})}} x: x \in \mathcal{N}_{f, \varphi, \mathcal{I}} \otimes \mathcal{D}_{C_{f, \varphi, T}^{*}}\right\}
$$

Consequently, we obtain $z=0$. This shows that

$$
\widetilde{\mathcal{H}}=\left\{P_{\widetilde{\mathcal{H}}}(y \oplus 0): y \in \mathcal{N}_{f, \varphi, \mathcal{I}} \otimes \mathcal{D}_{C_{f, \varphi, T}}\right\}^{-}
$$

Applying (3.12) and (3.13), we deduce that there exists a unique unitary operator $W: \mathcal{H} \rightarrow$ $\widetilde{\mathcal{H}}$ such that

$$
W\left(K_{f, \varphi, T}^{(\mathcal{I})} y\right)=P_{\widetilde{\mathcal{H}}}(y \oplus 0), \quad y \in \mathcal{N}_{f, \varphi, \mathcal{I}} \otimes \mathcal{D}_{C_{f, \varphi, T}} .
$$

Moreover, using (3.9) and Theorem 3.2 , we have

$$
\begin{aligned}
P_{\mathcal{N}_{f, \varphi, \mathcal{I}} \otimes \mathcal{D}_{C_{f, \varphi, T}} W\left(K_{f, \varphi, T}^{(\mathcal{I})}\right)^{*} y}=P_{\mathcal{N}_{f, \varphi, \mathcal{I} \otimes} \mathcal{D}_{C_{f, \varphi, T}} P_{\widetilde{\mathcal{H}}}(y \oplus 0)} & y-P_{\mathcal{N}_{f, \varphi, \mathcal{I}} \otimes \mathcal{D}_{C_{f, \varphi, T}}} \Psi \Psi^{*}(y \oplus 0) \\
& =y-\Theta_{f, \varphi, T}^{(\mathcal{I})}\left(\Theta_{f, \varphi, T}^{(\mathcal{I})}\right)^{*} y \\
& =K_{f, \varphi, T}^{(\mathcal{I})}\left(K_{f, \varphi, T}^{(\mathcal{I})}\right)^{*} y
\end{aligned}
$$

for any $y \in \mathcal{N}_{f, \varphi, \mathcal{I}} \otimes \mathcal{D}_{C_{f, \varphi, T}}$. Since the range $\left(K_{f, \varphi, T}^{(\mathcal{I})}\right)^{*}$ is dense in $\mathcal{H}$, we infer that

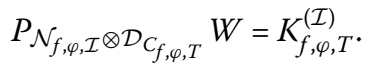

Let $\widetilde{T}_{i}: \widetilde{\mathcal{H}} \rightarrow \widetilde{\mathcal{H}}$ be the transform of $T_{i}$ under the unitary operator $W: \mathcal{H} \rightarrow \widetilde{\mathcal{H}}$, i.e.,

$$
\widetilde{T}_{i}=W T_{i} W^{*}, \quad i=1, \ldots, n
$$

Since the constrained Poisson kernel $K_{f, \varphi, T}^{(\mathcal{I})}$ is an injective operator, due to (3.14), we deduce that

$$
P_{\mathcal{N}_{f, \varphi, \mathcal{I}} \otimes \mathcal{D}_{C, \varphi, T}} \mid \tilde{\mathcal{H}}=K_{f, \varphi, T}^{(\mathcal{I})} W^{*}
$$


is an injective operator acting from $\widetilde{\mathcal{H}}$ to $\mathcal{N}_{f, \varphi, \mathcal{I}} \otimes \mathcal{D}_{C_{f, \varphi, T}}$. Consequently, according to (3.14) and Theorem 3.1, we have

$$
\begin{aligned}
\left(P_{\left.\mathcal{N}_{f, \varphi, \mathcal{I}} \otimes \mathcal{D}_{C_{f, \varphi}, T} \mid \widetilde{\mathcal{H}}\right)}\right) \widetilde{T}_{i}^{*} W h & =\left(P_{\mathcal{N}_{f, \varphi, \mathcal{I}} \otimes \mathcal{D}_{C_{f, \varphi, T}} \mid \widetilde{\mathcal{H}}}\right) W T_{i}^{*} h \\
& =K_{f, \varphi, T}^{(\mathcal{I})} T_{i}^{*} h \\
& =\left(B_{i}^{*} \otimes I_{\mathcal{D}_{C_{f, \varphi, T}}}\right) K_{f, \varphi, T}^{(\mathcal{I})} h \\
& =\left(B_{i}^{*} \otimes I_{\mathcal{D}_{C_{f, \varphi}, T}}\right)\left(P_{\mathcal{N}_{f, \varphi, \mathcal{I}} \otimes \mathcal{D}_{C_{f, \varphi, T}} \mid \widetilde{\mathcal{H}}}\right) W h
\end{aligned}
$$

for any $h \in \mathcal{H}$ and $i=1, \ldots, n$. Hence, we obtain that

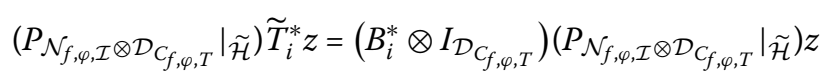

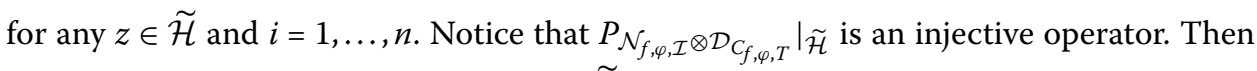
(3.15) uniquely determines each operator $\widetilde{T}_{i}, i=1, \ldots, n$.

(ii) First, assume that $T=\left(T_{1}, \ldots, T_{n}\right) \in \mathcal{V}_{f, \varphi, \mathcal{I}}^{\text {pure }}(\mathcal{H})$. Due to Theorem 3.1, we know that the constrained Poisson kernel $K_{f, \varphi, T}^{(\mathcal{I})}: \mathcal{H} \rightarrow \mathcal{N}_{f, \varphi, \mathcal{I}} \otimes \mathcal{D}_{C_{f, \varphi, T}}$ is an isometry. Hence, $K_{f, \varphi, T}^{(\mathcal{I})}\left(K_{f, \varphi, T}^{(\mathcal{I})}\right)^{*}$ is the orthogonal projection from $\mathcal{N}_{f, \varphi, \mathcal{I}} \otimes \mathcal{D}_{C_{f, \varphi, T}}$ onto $K_{f, \varphi, T}^{(\mathcal{I})} \mathcal{H}$. According to Theorem 3.2, we deduce that $\Theta_{f, \varphi, T}^{(\mathcal{I})}\left(\Theta_{f, \varphi, T}^{(\mathcal{I})}\right)^{*}$ is also a projection, which implies that $\Theta_{f, \varphi, T}^{(\mathcal{I})}$ is a partial isometry. This shows that $\Theta_{f, \varphi, T}^{(\mathcal{I})}$ is an inner multi-analytic operator.

Conversely, if $\Theta_{f, \varphi, T}^{(\mathcal{I})}$ is an inner multi-analytic operator, then it is a partial isometry. Applying Theorem 3.2, we infer that $K_{f, \varphi, T}^{(\mathcal{I})}$ is a partial isometry. Moreover, since $T$ is in the noncommutative variety $\mathcal{V}_{f, \varphi, \mathcal{I}}^{\mathrm{cnc}}(\mathcal{H})$, due to (3.5), we deduce that $K_{f, \varphi, T}^{(\mathcal{I})}$ is an injective operator, which implies that $K_{f, \varphi, T}^{(\mathcal{I})}$ is an isometry. Therefore, using Theorem 3.1, we deduce that $T$ is in $\mathcal{V}_{f, \varphi, \mathcal{I}}^{\text {pure }}(\mathcal{H})$.

Now, we prove the last part of the theorem. Notice that $u \oplus v \in \widetilde{\mathcal{K}}$ is in $\widetilde{\mathcal{H}}$ if and only if

$$
\left\langle u \oplus v, \Theta_{f, \varphi, T}^{(\mathcal{I})} x \oplus \Delta_{\Theta_{C_{f, \varphi, T}^{(\mathcal{I})}}} x\right\rangle=0
$$

for any $x \in \mathcal{N}_{f, \varphi, \mathcal{I}} \otimes \mathcal{D}_{C_{f, \varphi, T}^{*}}$. Note that condition (3.16) is equivalent to

$$
\left(\Theta_{f, \varphi, T}^{(\mathcal{I})}\right)^{*} u+\Delta_{\Theta_{f, \varphi, T}^{(\mathcal{I})}} v=0
$$

Since the operator $\Delta_{\Theta_{f, \varphi, T}^{(\mathcal{I})}}$ is the orthogonal projection from $\mathcal{N}_{f, \varphi, \mathcal{I}} \otimes \mathcal{D}_{C_{f, \varphi, T}^{*}}$ onto $\left[\operatorname{range}\left(\Theta_{f, \varphi, T}^{(\mathcal{I})}\right)^{*}\right]^{\perp}$, we have

$$
\left(\Theta_{f, \varphi, T}^{(\mathcal{I})}\right)^{*} u \perp \Delta_{\Theta_{f, \varphi, T}^{(\mathcal{I})} v} v
$$

Hence, (3.17) holds if and only if $\left(\Theta_{f, \varphi, T}^{(\mathcal{I})}\right)^{*} u=0$ and $v=0$. Therefore, we conclude that

$$
\widetilde{\mathcal{K}}=\mathcal{N}_{f, \varphi, \mathcal{I}} \otimes \mathcal{D}_{C_{f, \varphi, T}}
$$

and

$$
\widetilde{\mathcal{H}}=\left(\mathcal{N}_{f, \varphi, \mathcal{I}} \otimes \mathcal{D}_{C_{f, \varphi}, T}\right) \ominus \Theta_{f, \varphi, T}^{(\mathcal{I})}\left(\mathcal{N}_{f, \varphi, \mathcal{I}} \otimes \mathcal{D}_{C_{f, \varphi, T}^{*}}\right) .
$$


According to (3.15), we infer that

$$
\widetilde{T}_{i}=\left.P_{\widetilde{\mathcal{H}}}\left(B_{i} \otimes I_{\mathcal{D}_{C_{f, \varphi}, T}}\right)\right|_{\widetilde{\mathcal{H}}}, \quad i=1, \ldots, n
$$

This completes the proof.

Let $\Phi: \mathcal{N}_{f, \varphi, \mathcal{I}} \otimes \mathcal{H}_{1} \rightarrow \mathcal{N}_{f, \varphi, \mathcal{I}} \otimes \mathcal{H}_{2}$ and $\Phi^{\prime}: \mathcal{N}_{f, \varphi, \mathcal{I}} \otimes \mathcal{H}_{1}^{\prime} \rightarrow \mathcal{N}_{f, \varphi, \mathcal{I}} \otimes \mathcal{H}_{2}^{\prime}$ be two multianalytic operators with respect to the constrained weighted shifts $B_{1}, \ldots, B_{n}$, i.e.,

$$
\Phi\left(B_{i} \otimes I_{\mathcal{H}_{1}}\right)=\left(B_{i} \otimes I_{\mathcal{H}_{2}}\right) \Phi \quad \text { and } \quad \Phi^{\prime}\left(B_{i} \otimes I_{\mathcal{H}_{1}^{\prime}}\right)=\left(B_{i} \otimes I_{\mathcal{H}_{2}^{\prime}}\right) \Phi^{\prime}
$$

for any $i=1, \ldots, n$. We say that $\Phi$ and $\Phi^{\prime}$ coincide if there exist two unitary operators $U_{j} \in B\left(\mathcal{H}_{j}, \mathcal{H}_{j}^{\prime}\right), j=1,2$, such that

$$
\Phi^{\prime}\left(I_{\mathcal{N}_{f, \varphi, \mathcal{I}}} \otimes U_{1}\right)=\left(I_{\mathcal{N}_{f, \varphi, \mathcal{I}}} \otimes U_{2}\right) \Phi
$$

Applying Theorem 3.3, we can show that the constrained characteristic function $\Theta_{f, \varphi, T}^{(\mathcal{I})}$ is a complete unitary invariant for the $n$-tuples of operators in the noncommutative variety $\mathcal{V}_{f, \varphi, \mathcal{I}}^{\mathrm{cnc}}(\mathcal{H})$.

Theorem 3.4 Let $f:=\sum_{\alpha \in \mathbb{F}_{n}^{+}} a_{\alpha} Z_{\alpha}$ be a positive regular free holomorphic function, and let $\varphi=\left(\varphi_{1}, \ldots, \varphi_{n}\right)$ be an $n$-tuple offormal power series with model property. Let $\mathcal{I} \neq H^{\infty}\left(\mathbb{D}_{f, \varphi}\right)$ be a WOT-closed two-sided ideal of the noncommutative Hardy algebra $H^{\infty}\left(\mathbb{D}_{f, \varphi}\right)$. If $T=\left(T_{1}, \ldots, T_{n}\right) \in \mathcal{V}_{f, \varphi, \mathcal{I}}^{\mathrm{cnc}}(\mathcal{H})$ and $T^{\prime}=\left(T_{1}^{\prime}, \ldots, T_{n}^{\prime}\right) \in \mathcal{V}_{f, \varphi, \mathcal{I}}^{\mathrm{cnc}}\left(\mathcal{H}^{\prime}\right)$, then $T$ and $T^{\prime}$ are unitarily equivalent if and only if their constrained characteristic functions $\Theta_{f, \varphi, T}^{(\mathcal{I})}$ and $\Theta_{f, \varphi, T^{\prime}}^{(\mathcal{I})}$ coincide.

Proof First, we assume that $\Theta_{f, \varphi, T}^{(\mathcal{I})}$ and $\Theta_{f, \varphi, T^{\prime}}^{(\mathcal{I})}$ coincide. Then there are two unitary operators $U_{1}: \mathcal{D}_{C_{f, \varphi, T}} \rightarrow \mathcal{D}_{C_{f, \varphi, T^{\prime}}}$ and $U_{2}: \mathcal{D}_{C_{f, \varphi, T}^{*}} \rightarrow \mathcal{D}_{C_{f, \varphi, T^{\prime}}^{*}}$ such that

$$
\left(I_{\mathcal{N}_{f, \varphi, \mathcal{I}}} \otimes U_{1}\right) \Theta_{f, \varphi, T}^{(\mathcal{I})}=\Theta_{f, \varphi, T^{\prime}}^{(\mathcal{I})}\left(I_{\mathcal{N}_{f, \varphi, \mathcal{I}}} \otimes U_{2}\right)
$$

Consequently, we have

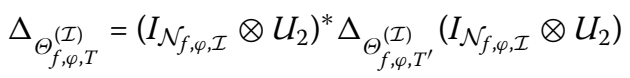

and

$$
\left(I_{\mathcal{N}_{f, \varphi, \mathcal{I}}} \otimes U_{2}\right) \overline{\left[\Delta_{\Theta_{f, \varphi, T}^{(\mathcal{I})}}\left(\mathcal{N}_{f, \varphi, \mathcal{I}} \otimes \mathcal{D}_{C_{f, \varphi, T}^{*}}\right)\right]}=\overline{\left[\Delta_{\left.\Theta_{f, \varphi, T^{\prime}}^{(\mathcal{I})}\left(\mathcal{N}_{f, \varphi, \mathcal{I}} \otimes \mathcal{D}_{C_{f, \varphi, T^{\prime}}^{*}}\right)\right]} .\right.}
$$

Now we define the unitary operator $W: \widetilde{\mathcal{K}} \rightarrow \widetilde{\mathcal{K}}^{\prime}$ by setting

$$
W:=\left(I_{\mathcal{N}_{f, \varphi, \mathcal{I}}} \otimes U_{1}\right) \oplus\left(I_{\mathcal{N}_{f, \varphi, \mathcal{I}}} \otimes U_{2}\right)
$$

where $\widetilde{\mathcal{K}}$ and $\widetilde{\mathcal{K}}^{\prime}$ were defined in Theorem 3.3. Notice that the operator $\Psi: \mathcal{N}_{f, \varphi, \mathcal{I}} \otimes$ $\mathcal{D}_{C_{f, \varphi, T}^{*}} \rightarrow \widetilde{\mathcal{K}}$, defined by

$$
\Psi x:=\Theta_{f, \varphi, T}^{(\mathcal{I})} x \oplus \Delta_{\Theta_{f, \varphi, T}^{(\mathcal{I})}} x, \quad x \in \mathcal{N}_{f, \varphi, \mathcal{I}} \otimes \mathcal{D}_{C_{f, \varphi, T}^{*}},
$$


and the corresponding $\Psi^{\prime}: \mathcal{N}_{f, \varphi, \mathcal{I}} \otimes \mathcal{D}_{C_{f, \varphi, T^{\prime}}^{*}} \rightarrow \widetilde{\mathcal{K}}^{\prime}$ satisfy the following relations:

$$
W \Psi\left(I_{\mathcal{N}_{f, \varphi, \mathcal{I}}} \otimes U_{2}\right)^{*}=\Psi^{\prime}
$$

and

$$
\left(I_{\mathcal{N}_{f, \varphi, \mathcal{I}}} \otimes U_{1}\right) P_{\mathcal{N}_{f, \varphi, \mathcal{I}} \otimes \mathcal{D}_{C_{f, \varphi, T}}}^{\tilde{\mathcal{N}}} W^{*}=P_{\mathcal{N}_{f, \varphi, \mathcal{I}} \otimes \mathcal{D}_{C_{f, \varphi, T^{\prime}}}}^{\tilde{\mathcal{K}}^{\prime}}
$$

where $P_{\mathcal{N}_{f, \varphi, \mathcal{I}} \otimes \mathcal{D}_{C f, \varphi, T}}^{\widetilde{\mathcal{C}}}$ is the orthogonal projection from $\widetilde{\mathcal{K}}$ onto $\mathcal{N}_{f, \varphi, \mathcal{I}} \otimes \mathcal{D}_{C_{f, \varphi, T}}$. Hence, we have

$$
\begin{aligned}
W \widetilde{\mathcal{H}} & =W \widetilde{\mathcal{K}} \ominus W \Psi\left(\mathcal{N}_{f, \varphi, \mathcal{I}} \otimes \mathcal{D}_{C_{f, \varphi, T}^{*}}\right) \\
& =\widetilde{\mathcal{K}}^{\prime} \ominus \Psi^{\prime}\left(I_{\mathcal{N}_{f, \varphi}, \mathcal{I}} \otimes U_{2}\right)\left(\mathcal{N}_{f, \varphi, \mathcal{I}} \otimes \mathcal{D}_{C_{f, \varphi, T}^{*}}\right) \\
& =\widetilde{\mathcal{K}}^{\prime} \ominus \Psi^{\prime}\left(\mathcal{N}_{f, \varphi, \mathcal{I}} \otimes \mathcal{D}_{C_{f, \varphi, T^{\prime}}^{*}}\right) \\
& =\widetilde{\mathcal{H}}^{\prime}
\end{aligned}
$$

which implies that $\left.W\right|_{\tilde{\mathcal{H}}}: \widetilde{\mathcal{H}} \rightarrow \widetilde{\mathcal{H}}^{\prime}$ is unitary. On the other hand, for any $i=1, \ldots, n$,

$$
\left(B_{i}^{*} \otimes I_{\mathcal{D}_{C_{f, \varphi, T^{\prime}}}}\right)\left(I_{\mathcal{N}_{f, \varphi, \mathcal{I}}} \otimes U_{1}\right)=\left(I_{\mathcal{N}_{f, \varphi, \mathcal{I}}} \otimes U_{1}\right)\left(B_{i}^{*} \otimes I_{\mathcal{D}_{C_{f, \varphi, T}}}\right) .
$$

Now, we assume that $\widetilde{T}:=\left(\widetilde{T}_{1}, \ldots, \widetilde{T}_{n}\right)$ and $\widetilde{T}^{\prime}:=\left(\widetilde{T}_{1}^{\prime}, \ldots, \widetilde{T}_{n}^{\prime}\right)$ are the model operators provided by Theorem 3.3 for $T$ and $T^{\prime}$, respectively. Therefore, applying (3.18), (3.19), and (3.20), we deduce that

$$
\begin{aligned}
& P_{\mathcal{N}_{f, \varphi}, \mathcal{I} \otimes \mathcal{D}_{C_{f, \varphi}, T^{\prime}}}^{\widetilde{\widetilde{T}}_{i}^{\prime}} \widetilde{T}_{i}^{\prime *} W z=\left(B_{i}^{*} \otimes I_{\mathcal{D}_{C_{f, \varphi, T^{\prime}}}}\right) P_{\mathcal{N}_{f, \varphi, \mathcal{I}} \otimes \mathcal{D}_{C_{f, \varphi, T^{\prime}}}}^{{\widetilde{J^{\prime}}}^{\prime}} W z \\
& =\left(B_{i}^{*} \otimes I_{\mathcal{D}_{C_{f, \varphi, T^{\prime}}}}\right)\left(I_{\mathcal{N}_{f, \varphi, \mathcal{I}}} \otimes U_{1}\right) P_{\mathcal{N}_{f, \varphi, \mathcal{I}} \otimes \mathcal{D}_{C, \varphi, T}}^{\widetilde{\mathcal{K}}} z \\
& =\left(I_{\mathcal{N}_{f, \varphi, \mathcal{I}}} \otimes U_{1}\right)\left(B_{i}^{*} \otimes I_{\mathcal{D}_{C_{f, \varphi}, T}}\right) P_{\mathcal{N}_{f, \varphi, \mathcal{I}} \widetilde{\mathcal{L}}_{\mathcal{D}_{C f, \varphi}}} z \\
& =\left(I_{\mathcal{N}_{f, \varphi, \mathcal{I}}} \otimes U_{1}\right) P_{\mathcal{N}_{f, \varphi, \mathcal{I}} \widetilde{\mathcal{K}} \otimes \mathcal{D}_{C_{f, \varphi, T}}} \widetilde{T}_{i}^{*} z \\
& =P_{\mathcal{N}_{f, \varphi, \mathcal{I}} \otimes \mathcal{D}_{C_{f, \varphi, T^{\prime}}}}^{\widetilde{\mathcal{N}}^{\prime}} W \widetilde{T}_{i}^{*} z
\end{aligned}
$$

for any $z \in \tilde{\mathcal{H}}$ and $i=1, \ldots, n$. Using the fact that $P_{\mathcal{N}_{f, \varphi, \mathcal{I}} \otimes \mathcal{D}_{C_{f, \varphi}, T^{\prime}}}^{\widetilde{\mathcal{L}}^{\prime}}$ is an injective operator, we infer that

$$
\left(\left.W\right|_{\tilde{\mathcal{H}}}\right) \widetilde{T}_{i}^{*}=\widetilde{T}_{i}^{\prime *}\left(\left.W\right|_{\tilde{\mathcal{H}}}\right), \quad i=1, \ldots, n
$$

Due to Theorem 3.3, it is obvious that $T$ and $T^{\prime}$ are unitarily equivalent.

$$
\text { Conversely, let } \Omega: \mathcal{H} \rightarrow \mathcal{H}^{\prime} \text { be a unitary operator such that }
$$

$$
T_{i}=\Omega^{*} T_{i}^{\prime} \Omega \quad \text { for any } i=1, \ldots, n
$$


Note that $T \in \mathcal{C}_{\varphi}^{\text {SOT }}(\mathcal{H})$ or $T \in \mathcal{C}_{\varphi}^{\text {rad }}(\mathcal{H})$ and similar relations hold for $T^{\prime}$. Then we obtain

$$
\Omega \Delta_{C_{f, \varphi, T}}=\Delta_{C_{f, \varphi, T^{\prime}}} \Omega \quad \text { and } \quad\left(\oplus_{i=1}^{n} \Omega\right) \Delta_{C_{f, \varphi, T}^{*}}=\Delta_{C_{f, \varphi, T^{\prime}}^{*}}\left(\oplus_{i=1}^{n} \Omega\right)
$$

Now we define the unitary operator by setting

$$
U_{3}:=\left.\Omega\right|_{\mathcal{D}_{C_{f, \varphi}, T}}: \mathcal{D}_{C_{f, \varphi, T}} \rightarrow \mathcal{D}_{C_{f, \varphi, T^{\prime}}}
$$

and

$$
U_{4}:=\left.\left(\oplus_{i=1}^{n} \Omega\right)\right|_{\mathcal{D}_{C_{f, \varphi, T}^{*}}}: \mathcal{D}_{C_{f, \varphi, T}^{*}} \rightarrow \mathcal{D}_{C_{f, \varphi, T^{\prime}}^{*}}
$$

A simple calculation shows that

$$
\left(I_{\mathcal{N}_{f, \varphi, \mathcal{I}}} \otimes U_{3}\right) \Theta_{f, \varphi, T}^{(\mathcal{I})}=\Theta_{f, \varphi, T^{\prime}}^{(\mathcal{I})}\left(I_{\mathcal{N}_{f, \varphi, \mathcal{I}}} \otimes U_{4}\right)
$$

This completes the proof.

\section{Multivariable interpolation and invariant subspaces}

In this section, we prove a Sarason-type commutant lifting theorem. As an application, we obtain the Nevanlinna-Pick-type interpolation result in our setting. Moreover, we provide a Beurling-type characterization of the joint invariant subspaces under the constrained weighted shifts $B_{1}, \ldots, B_{n}$.

For each $i=1, \ldots, n$, we define the right multiplication operator $R_{i}: \mathcal{F}_{f}^{2} \rightarrow \mathcal{F}_{f}^{2}$ by setting $R_{i} \zeta=\zeta Z_{i}, \zeta \in \mathcal{F}_{f}^{2}$. Using the results from [24], we know that $R^{\infty}\left(\mathcal{D}_{f}\right)$ is the WOT-closure of all polynomials in $R_{1}, \ldots, R_{n}$ and the identity. Moreover, we define the noncommutative Hardy algebra $R^{\infty}\left(\mathbb{D}_{f, \varphi}\right)$ to be the WOT-closure of all noncommutative polynomials in $R_{Z_{1}}, \ldots, R_{Z_{n}}$ and the identity.

The following result is a Sarason-type [32] commutant lifting theorem.

Theorem 4.1 Letf $:=\sum_{\alpha \in \mathbb{F}_{n}^{+}} a_{\alpha} Z_{\alpha}$ be a positive regular free holomorphic function, and let $\varphi=\left(\varphi_{1}, \ldots, \varphi_{n}\right)$ be an $n$-tuple offormal power series with model property. Let $\mathcal{I} \neq H^{\infty}\left(\mathbb{D}_{f, \varphi}\right)$ be a WOT-closed two-sided ideal of the noncommutative Hardy algebra $H^{\infty}\left(\mathbb{D}_{f, \varphi}\right)$. For each $j=1,2$, let $\mathcal{K}_{j}$ be a Hilbert space, and let $\mathcal{E}_{j} \subseteq \mathcal{N}_{f, \varphi, \mathcal{I}} \otimes \mathcal{K}_{j}$ be an invariant subspace under $B_{i}^{*} \otimes I_{\mathcal{K}_{j}}, i=1, \ldots, n$. If $X: \mathcal{E}_{1} \rightarrow \mathcal{E}_{2}$ is a bounded operator such that

$$
X\left[P_{\mathcal{E}_{1}}\left(B_{i} \otimes I_{\mathcal{K}_{1}}\right) \mid \mathcal{E}_{1}\right]=\left[\left.P_{\mathcal{E}_{2}}\left(B_{i} \otimes I_{\mathcal{K}_{2}}\right)\right|_{\mathcal{E}_{2}}\right] X, \quad i=1, \ldots, n,
$$

then there exists

$$
\Phi\left(C_{1}, \ldots, C_{n}\right) \in R^{\infty}\left(\mathcal{V}_{f, \varphi, \mathcal{I}}\right) \bar{\otimes} B\left(\mathcal{K}_{1}, \mathcal{K}_{2}\right)
$$

such that

$$
\Phi\left(C_{1}, \ldots, C_{n}\right)^{*} \mathcal{E}_{2} \subseteq \mathcal{E}_{1},\left.\quad \Phi\left(C_{1}, \ldots, C_{n}\right)^{*}\right|_{\mathcal{E}_{2}}=X^{*}, \quad \text { and } \quad\left\|\Phi\left(C_{1}, \ldots, C_{n}\right)\right\|=\|X\|
$$


Proof First, note that the subspace $\mathcal{N}_{f, \varphi, \mathcal{I}} \otimes \mathcal{K}_{j}$ is invariant under $M_{Z_{i}}^{*} \otimes I_{\mathcal{K}_{j}}$, and

$$
\left.\left(M_{Z_{i}}^{*} \otimes I_{\mathcal{K}_{j}}\right)\right|_{\mathcal{N}_{f, \varphi, \mathcal{I}} \otimes \mathcal{K}_{j}}=B_{i}^{*} \otimes I_{\mathcal{K}_{j}}, \quad i=1, \ldots, n
$$

Since $\mathcal{E}_{j} \subseteq \mathcal{N}_{f, \varphi, \mathcal{I}} \otimes \mathcal{K}_{j}$ is invariant under $B_{1}^{*} \otimes I_{\mathcal{K}_{j}}, \ldots, B_{n}^{*} \otimes I_{\mathcal{K}_{j}}$, it is also invariant under $M_{Z_{1}}^{*} \otimes I_{\mathcal{K}_{j}}, \ldots, M_{Z_{n}}^{*} \otimes I_{\mathcal{K}_{j}}$, which implies that

$$
\left.\left(M_{Z_{i}}^{*} \otimes I_{\mathcal{K}_{j}}\right)\right|_{\mathcal{E}_{j}}=\left.\left(B_{i}^{*} \otimes I_{\mathcal{K}_{j}}\right)\right|_{\mathcal{E}_{j}}, \quad i=1, \ldots, n
$$

Hence, we deduce that

$$
X\left[\left.P_{\mathcal{E}_{1}}\left(M_{Z_{i}} \otimes I_{\mathcal{K}_{1}}\right)\right|_{\mathcal{E}_{1}}\right]=\left[\left.P_{\mathcal{E}_{2}}\left(M_{Z_{i}} \otimes I_{\mathcal{K}_{2}}\right)\right|_{\mathcal{E}_{2}}\right] X, \quad i=1, \ldots, n .
$$

According to Theorem 5.1 of [28], there exists a bounded operator $\Phi: \mathbb{H}_{f}^{2}(\varphi) \otimes \mathcal{K}_{1} \rightarrow$ $\mathbb{H}_{f}^{2}(\varphi) \otimes \mathcal{K}_{2}$ with the property

$$
\Phi\left(M_{Z_{i}} \otimes I_{\mathcal{K}_{1}}\right)=\left(M_{Z_{i}} \otimes I_{\mathcal{K}_{2}}\right) \Phi, \quad i=1, \ldots, n,
$$

and such that $\Phi^{*} \mathcal{E}_{2} \subseteq \mathcal{E}_{1},\left.\Phi^{*}\right|_{\mathcal{E}_{2}}=X^{*}$, and $\|\Phi\|=\|X\|$. Since $M_{\varphi_{i}}=\varphi_{i}\left(M_{Z_{1}}, \ldots, M_{Z_{n}}\right)$ for any $i=1, \ldots, n$, we have

$$
\Phi\left(M_{\varphi_{i}} \otimes I_{\mathcal{K}_{1}}\right)=\left(M_{\varphi_{i}} \otimes I_{\mathcal{K}_{2}}\right) \Phi, \quad i=1, \ldots, n
$$

Notice that

$$
M_{\varphi_{i}}=U^{-1} V_{i} U, \quad i=1, \ldots, n .
$$

Then we obtain

$$
\Phi\left(U^{-1} \otimes I_{\mathcal{K}_{1}}\right)\left(V_{i} \otimes I_{\mathcal{K}_{1}}\right)\left(U \otimes I_{\mathcal{K}_{1}}\right)=\left(U^{-1} \otimes I_{\mathcal{K}_{2}}\right)\left(V_{i} \otimes I_{\mathcal{K}_{2}}\right)\left(U \otimes I_{\mathcal{K}_{2}}\right) \Phi
$$

for any $i=1, \ldots, n$. This shows that

$$
\left[\left(U \otimes I_{\mathcal{K}_{2}}\right) \Phi\left(U^{-1} \otimes I_{\mathcal{K}_{1}}\right)\right]\left(V_{i} \otimes I_{\mathcal{K}_{1}}\right)=\left(V_{i} \otimes I_{\mathcal{K}_{2}}\right)\left[\left(U \otimes I_{\mathcal{K}_{2}}\right) \Phi\left(U^{-1} \otimes I_{\mathcal{K}_{1}}\right)\right]
$$

for any $i=1, \ldots, n$. Due to the discussion of Proposition 1.11 from [24], we infer that

$$
\left[\left(U \otimes I_{\mathcal{K}_{2}}\right) \Phi\left(U^{-1} \otimes I_{\mathcal{K}_{1}}\right)\right] \in R^{\infty}\left(\mathcal{D}_{f}\right) \bar{\otimes} B\left(\mathcal{K}_{1}, \mathcal{K}_{2}\right)
$$

Using Proposition 4.2 in [28], we know

$$
R^{\infty}\left(\mathbb{D}_{f, \varphi}\right)=U^{-1} R^{\infty}\left(\mathcal{D}_{f}\right) U
$$

Consequently, we infer that

$$
\Phi \in R^{\infty}\left(\mathbb{D}_{f, \varphi}\right) \bar{\otimes} B\left(\mathcal{K}_{1}, \mathcal{K}_{2}\right)
$$


Assume that $\Phi\left(R_{Z_{1}}, \ldots, R_{Z_{n}}\right):=\Phi$. This shows that we can find $\Phi\left(R_{Z_{1}}, \ldots, R_{Z_{n}}\right) \in$ $R^{\infty}\left(\mathbb{D}_{f, \varphi}\right) \bar{\otimes} B\left(\mathcal{K}_{1}, \mathcal{K}_{2}\right)$ such that $\Phi\left(R_{Z_{1}}, \ldots, R_{Z_{n}}\right)^{*} \mathcal{E}_{2} \subseteq \mathcal{E}_{1}$,

$$
\left.\Phi\left(R_{Z_{1}}, \ldots, R_{Z_{n}}\right)^{*}\right|_{\mathcal{E}_{2}}=X^{*} \text { and }\left\|\Phi\left(R_{Z_{1}}, \ldots, R_{Z_{n}}\right)\right\|=\|X\|
$$

Moreover, we assume that

$$
\Phi\left(C_{1}, \ldots, C_{n}\right):=\left.P_{\mathcal{N}_{f, \varphi, \mathcal{I}} \otimes \mathcal{K}_{2}} \Phi\left(R_{Z_{1}}, \ldots, R_{Z_{n}}\right)\right|_{\mathcal{N}_{f, \varphi, \mathcal{I}} \otimes \mathcal{K}_{1}}
$$

Then we have $\Phi\left(C_{1}, \ldots, C_{n}\right) \in R^{\infty}\left(\mathcal{V}_{f, \varphi, \mathcal{I}}\right) \bar{\otimes} B\left(\mathcal{K}_{1}, \mathcal{K}_{2}\right)$. Notice that

$$
\Phi\left(R_{Z_{1}}, \ldots, R_{Z_{n}}\right)^{*}\left(\mathcal{N}_{f, \varphi, \mathcal{I}} \otimes \mathcal{K}_{2}\right) \subseteq \mathcal{N}_{f, \varphi, \mathcal{I}} \otimes \mathcal{K}_{1}
$$

and $\mathcal{E}_{j} \subseteq \mathcal{N}_{f, \varphi, \mathcal{I}} \otimes \mathcal{K}_{j}$. Using (4.2), we obtain

$$
\Phi\left(C_{1}, \ldots, C_{n}\right)^{*} \mathcal{E}_{2} \subseteq \mathcal{E}_{1} \quad \text { and }\left.\quad \Phi\left(C_{1}, \ldots, C_{n}\right)^{*}\right|_{\mathcal{E}_{2}}=X^{*}
$$

Applying again (4.2), we infer that

$$
\|X\| \leq\left\|\Phi\left(C_{1}, \ldots, C_{n}\right)\right\| \leq\left\|\Phi\left(R_{Z_{1}}, \ldots, R_{Z_{n}}\right)\right\|=\|X\|,
$$

which shows that

$$
\left\|\Phi\left(C_{1}, \ldots, C_{n}\right)\right\|=\|X\| \text {. }
$$

This completes the proof.

Applying Theorem 4.1, we can obtain the following Nevanlinna-Pick-type interpolation result in our setting.

Theorem 4.2 Let $f:=\sum_{\alpha \in \mathbb{F}_{n}^{+}} a_{\alpha} Z_{\alpha}$ be a positive regular free holomorphic function, and let $\varphi=\left(\varphi_{1}, \ldots, \varphi_{n}\right)$ be an $n$-tuple offormal power series with model property. Let $\mathcal{I} \neq H^{\infty}\left(\mathbb{D}_{f, \varphi}\right)$ be a WOT-closed two-sided ideal of the noncommutative Hardy algebra $H^{\infty}\left(\mathbb{D}_{f, \varphi}\right)$. Let $\lambda_{1}, \ldots, \lambda_{k}$ be $k$ distinct points in $\mathcal{V}_{f, \varphi, \mathcal{I}}^{<}(\mathbb{C})$, and let $A_{1}, \ldots, A_{k} \in B(\mathcal{K})$. Then there exists $\Phi\left(C_{1}, \ldots, C_{n}\right) \in R^{\infty}\left(\mathcal{V}_{f, \varphi, \mathcal{I}}\right) \bar{\otimes} B(\mathcal{K})$ such that

$$
\left\|\Phi\left(C_{1}, \ldots, C_{n}\right)\right\| \leq 1 \quad \text { and } \quad \Phi\left(\lambda_{j}\right)=A_{j}, \quad j=1, \ldots, k
$$

if and only if the operator matrix

$$
\left[K_{f, \varphi}\left(\lambda_{i}, \lambda_{j}\right)\left(I_{\mathcal{K}}-A_{i} A_{j}^{*}\right)\right]_{k \times k}
$$

is positive semidefinite, where

$$
K_{f, \varphi}\left(\lambda_{i}, \lambda_{j}\right):=\frac{\sqrt{1-\sum_{|\alpha| \geq 1} a_{\alpha}\left|\varphi_{\alpha}\left(\lambda_{i}\right)\right|^{2}} \sqrt{1-\sum_{|\alpha| \geq 1} a_{\alpha}\left|\varphi_{\alpha}\left(\lambda_{j}\right)\right|^{2}}}{1-\sum_{|\alpha| \geq 1} a_{\alpha}\left[\varphi\left(\lambda_{i}\right)\right]_{\alpha}\left[\overline{\varphi\left(\lambda_{j}\right)}\right]_{\alpha}} .
$$


Proof Let $\lambda_{j}:=\left(\lambda_{j_{1}}, \ldots, \lambda_{j_{n}}\right), j=1, \ldots, k$, be $k$ distinct points in $\mathcal{V}_{f, \varphi, \mathcal{I}}^{<}(\mathbb{C})$, and let

$$
Z_{f, \varphi}^{\left(\lambda_{j}\right)}:=\sqrt{1-\sum_{|\alpha| \geq 1} a_{\alpha}\left|\varphi_{\alpha}\left(\lambda_{j}\right)\right|^{2}}\left(\sum_{\alpha \in \mathbb{F}_{n}^{+}} b_{\alpha}\left[\overline{\varphi\left(\lambda_{j}\right)}\right]_{\alpha} \varphi_{\alpha}\right), \quad j=1, \ldots, k
$$

where the coefficients $b_{\alpha}, \alpha \in \mathbb{F}_{n}^{+}$, are given by relation (2.1). Since $\varphi$ has model property, we have

$$
M_{\varphi_{i}}=\varphi_{i}\left(M_{Z_{1}}, \ldots, M_{Z_{n}}\right), \quad i=1, \ldots, n,
$$

where $\left(M_{Z_{1}}, \ldots, M_{Z_{n}}\right)$ is either in the set $\mathcal{C}_{\varphi}^{\mathrm{SOT}}\left(\mathbb{H}_{f}^{2}(\varphi)\right)$ or $\mathcal{C}_{\varphi}^{\mathrm{rad}}\left(\mathbb{H}_{f}^{2}(\varphi)\right)$. Due to Proposition 4.2 of [28], for any $\omega \in \mathcal{I} \subseteq H^{\infty}\left(\mathbb{D}_{f, \varphi}\right)$, there exists $\chi=\sum_{\alpha \in \mathbb{F}_{n}^{+}} c_{\alpha} V_{\alpha} \in F^{\infty}\left(\mathcal{D}_{f}\right)$ such that

$$
\omega=\mathrm{SOT}-\lim _{r \rightarrow 1} \sum_{k=0}^{\infty} \sum_{|\alpha|=k} c_{\alpha} r^{|\alpha|} M_{\varphi_{\alpha}}
$$

Using (4.4) and (4.5), we infer that

$$
\left\langle Z_{f, \varphi}^{\left(\lambda_{j}\right)}, \omega(1)\right\rangle_{f, \varphi}=0 \quad \text { for any } \omega \in \mathcal{I} \text { and } j=1, \ldots, k
$$

Since $\mathcal{I}$ is a WOT-closed two-sided ideal of $H^{\infty}\left(\mathbb{D}_{f, \varphi}\right)$, we obtain

$$
\mathcal{M}_{f, \varphi, \mathcal{I}}=\overline{\mathcal{I}(1)}
$$

This shows that

$$
Z_{f, \varphi}^{\left(\lambda_{j}\right)} \in \mathcal{N}_{f, \varphi, \mathcal{I}}, \quad j=1, \ldots, k
$$

According to Theorem 4.4 of [28], we have

$$
M_{Z_{i}}^{*} Z_{f, \varphi}^{\left(\lambda_{j}\right)}=\overline{\lambda_{j i}} Z_{f, \varphi}^{\left(\lambda_{j}\right)}, \quad i=1, \ldots, n ; j=1, \ldots, k
$$

Moreover, notice that

$$
\left.B_{i}^{*}\right|_{\mathcal{N}_{f, \varphi, \mathcal{I}}}=\left.M_{Z_{i}}^{*}\right|_{\mathcal{N}_{f, \varphi, \mathcal{I}}}, \quad i=1, \ldots, n
$$

Hence, we deduce that the subspace

$$
\mathcal{M}:=\operatorname{span}\left\{Z_{f, \varphi}^{\left(\lambda_{j}\right)}: j=1, \ldots, k\right\}
$$

is invariant under $B_{i}^{*}$ for any $i=1, \ldots, n$, and $\mathcal{M} \subseteq \mathcal{N}_{f, \varphi, \mathcal{I}}$. Now, we define the operators $X_{i} \in B(\mathcal{M} \otimes \mathcal{K})$ by setting

$$
X_{i}:=\left.P_{\mathcal{M}} B_{i}\right|_{\mathcal{M}} \otimes I_{\mathcal{K}}, \quad i=1, \ldots, n
$$


Note that $Z_{f, \varphi}^{\left(\lambda_{1}\right)}, \ldots, Z_{f, \varphi}^{\left(\lambda_{k}\right)}$ are linearly independent. Then we can define an operator $T \in$ $B(\mathcal{M} \otimes \mathcal{K})$ by setting

$$
T^{*}\left(Z_{f, \varphi}^{\left(\lambda_{j}\right)} \otimes h\right)=Z_{f, \varphi}^{\left(\lambda_{j}\right)} \otimes A_{j}^{*} h
$$

for any $h \in \mathcal{K}$ and $j=1, \ldots, k$. A simple calculation shows that

$$
T X_{i}=X_{i} T, \quad i=1, \ldots, n
$$

Taking into account that $\mathcal{M} \otimes \mathcal{K}$ is a co-invariant subspace under $B_{i} \otimes I_{\mathcal{K}}, i=1, \ldots, n$. Due to Theorem 4.1, we can find $\Phi\left(R_{Z_{1}}, \ldots, R_{Z_{n}}\right) \in R^{\infty}\left(\mathbb{D}_{f, \varphi}\right) \bar{\otimes} B(\mathcal{K})$ such that

$$
\Phi\left(C_{1}, \ldots, C_{n}\right):=\left.P_{\mathcal{N}_{f, \varphi, \mathcal{I}} \otimes \mathcal{K}} \Phi\left(R_{Z_{1}}, \ldots, R_{Z_{n}}\right)\right|_{\mathcal{N}_{f, \varphi, \mathcal{I}} \otimes \mathcal{K}} \in R^{\infty}\left(\mathcal{V}_{f, \varphi, \mathcal{I}}\right) \bar{\otimes} B(\mathcal{K})
$$

has the properties

$$
\Phi\left(C_{1}, \ldots, C_{n}\right)^{*}(\mathcal{M} \otimes \mathcal{K}) \subseteq \mathcal{M} \otimes \mathcal{K},\left.\quad \Phi\left(C_{1}, \ldots, C_{n}\right)^{*}\right|_{\mathcal{M} \otimes \mathcal{K}}=T^{*}
$$

and

$$
\left\|\Phi\left(C_{1}, \ldots, C_{n}\right)\right\|=\|T\| .
$$

In what follows, we prove

$$
R_{Z_{i}}^{*} Z_{f, \varphi}^{(\lambda)}=\bar{\lambda}_{i} Z_{f, \varphi}^{(\lambda)} \quad \text { for any } \lambda \in \mathbb{D}_{f, \varphi}^{<}(\mathbb{C}) \text { and } i=1, \ldots, n,
$$

where $Z_{f, \varphi}^{(\lambda)}$ is given by relation (4.4). Indeed, a straightforward computation reveals that

$$
R_{\varphi_{\beta}}^{*} \varphi_{\alpha}= \begin{cases}\frac{b_{\gamma}}{b_{\alpha}} \varphi_{\gamma}, & \alpha=\gamma \widetilde{\beta} \\ 0, & \text { otherwise. }\end{cases}
$$

Consequently, we obtain

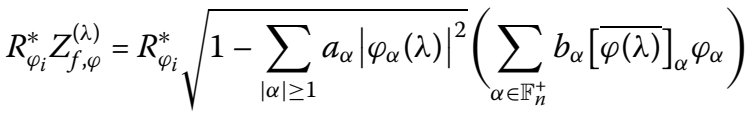

$$
\begin{aligned}
& =\sqrt{1-\sum_{|\alpha| \geq 1} a_{\alpha}\left|\varphi_{\alpha}(\lambda)\right|^{2}}\left(\sum_{\gamma \in \mathbb{F}_{n}^{+}} \frac{b_{\gamma}}{b_{\gamma g_{i}}} b_{\gamma g_{i}} \overline{[\varphi(\lambda)]_{\gamma g_{i}}} \varphi_{\gamma}\right) \\
& =\sqrt{1-\sum_{|\alpha| \geq 1} a_{\alpha}\left|\varphi_{\alpha}(\lambda)\right|^{2}}\left(\sum_{\gamma \in \mathbb{F}_{n}^{+}} b_{\gamma}{\overline{[\varphi(\lambda)]_{\gamma g_{i}}}}_{\left.\varphi_{\gamma}\right)}\right. \\
& =\overline{\varphi_{i}(\lambda)}\left[\sqrt{1-\sum_{|\alpha| \geq 1} a_{\alpha}\left|\varphi_{\alpha}(\lambda)\right|^{2}}\left(\sum_{\gamma \in \mathbb{F}_{n}^{+}} b_{\gamma} \overline{[\varphi(\lambda)]_{\gamma}} \varphi_{\gamma}\right)\right] \\
& =\overline{\varphi_{i}(\lambda)} Z_{f, \varphi}^{(\lambda)}
\end{aligned}
$$


for any $i=1, \ldots, n$. Moreover, due to the proof of Theorem 2.1 from [28], we have

$$
R_{Z_{i}}=\psi_{i}\left(R_{\varphi_{1}}, \ldots, R_{\varphi_{n}}\right)=\text { SOT }-\lim _{r \rightarrow 1} \psi_{i}\left(r R_{\varphi_{1}}, \ldots, r R_{\varphi_{n}}\right)
$$

for any $i=1, \ldots, n$. Hence, we conclude that

$$
\psi_{i}\left(R_{\varphi_{1}}, \ldots, R_{\varphi_{n}}\right)^{*} Z_{f, \varphi}^{(\lambda)}=\overline{\psi_{i}(\varphi(\lambda))} Z_{f, \varphi}^{(\lambda)}
$$

for any $i=1, \ldots, n$. Since $\lambda \in \mathbb{D}_{f, \varphi}^{<}(\mathbb{C})$, we obtain $\lambda_{i}=\psi_{i}(\varphi(\lambda))$ for any $i=1, \ldots, n$. Therefore, we infer that

$$
R_{Z_{i}}^{*} Z_{f, \varphi}^{(\lambda)}=\psi_{i}\left(R_{\varphi_{1}}, \ldots, R_{\varphi_{n}}\right)^{*} Z_{f, \varphi}^{(\lambda)}=\overline{\psi_{i}(\varphi(\lambda))} Z_{f, \varphi}^{(\lambda)}=\overline{\lambda_{i}} Z_{f, \varphi}^{(\lambda)}
$$

for any $i=1, \ldots, n$. This proves our assertion. Since $\lambda_{1}, \ldots, \lambda_{k}$ are $k$ distinct points in $\mathcal{V}_{f, \varphi, \mathcal{I}}^{<}(\mathbb{C}) \subseteq \mathbb{D}_{f, \varphi}^{<}(\mathbb{C})$, we have $R_{Z_{i}}^{*} Z_{f, \varphi}^{\left(\lambda_{j}\right)}=\overline{\lambda_{j i}} Z_{f, \varphi}^{\left(\lambda_{j}\right)}, i=1, \ldots, n ; j=1, \ldots, k$. This shows that

$$
v\left(R_{Z_{1}}, \ldots, R_{Z_{n}}\right)^{*} Z_{f, \varphi}^{\left(\lambda_{j}\right)}=\overline{v\left(\lambda_{j}\right)} Z_{f, \varphi}^{\left(\lambda_{j}\right)}
$$

for any $v\left(R_{Z_{1}}, \ldots, R_{Z_{n}}\right) \in R^{\infty}\left(\mathbb{D}_{f, \varphi}\right)$. Hence, we deduce that

$$
\Phi\left(R_{Z_{1}}, \ldots, R_{Z_{n}}\right)^{*}\left(Z_{f, \varphi}^{\left(\lambda_{j}\right)} \otimes h\right)=Z_{f, \varphi}^{\left(\lambda_{j}\right)} \otimes \Phi\left(\lambda_{j}\right)^{*} h, \quad j=1, \ldots, k .
$$

Using (4.6), we obtain

$$
\begin{aligned}
\langle\Phi & \left.\left(C_{1}, \ldots, C_{n}\right)^{*}\left(Z_{f, \varphi}^{\left(\lambda_{j}\right)} \otimes x\right), Z_{f, \varphi}^{\left(\lambda_{j}\right)} \otimes y\right\rangle \\
& =\left\langle\Phi\left(R_{Z_{1}}, \ldots, R_{Z_{n}}\right)^{*}\left(Z_{f, \varphi}^{\left(\lambda_{j}\right)} \otimes x\right), Z_{f, \varphi}^{\left(\lambda_{j}\right)} \otimes y\right\rangle \\
& =\left\langle Z_{f, \varphi}^{\left(\lambda_{j}\right)} \otimes \Phi\left(\lambda_{j}\right)^{*} x, Z_{f, \varphi}^{\left(\lambda_{j}\right)} \otimes y\right\rangle \\
& =\left\langle Z_{f, \varphi}^{\left(\lambda_{j}\right)},\left.Z_{f, \varphi}^{\left(\lambda_{j}\right)}\right|_{f, \varphi} \mid \Phi\left(\lambda_{j}\right)^{*} x, y\right\rangle
\end{aligned}
$$

for any $x, y \in \mathcal{K}$ and $j=1, \ldots, k$. Moreover, notice that

$$
\left\langle T^{*}\left(Z_{f, \varphi}^{\left(\lambda_{j}\right)} \otimes x\right), Z_{f, \varphi}^{\left(\lambda_{j}\right)} \otimes y\right\rangle=\left\langle Z_{f, \varphi}^{\left(\lambda_{j}\right)}, Z_{f, \varphi}^{\left(\lambda_{j}\right)}\right\rangle_{f, \varphi}\left\langle A_{j}^{*} x, y\right\rangle
$$

for any $x, y \in \mathcal{K}$ and $j=1, \ldots, k$. Since $\varphi\left(\lambda_{1}\right), \ldots, \varphi\left(\lambda_{k}\right)$ are in the strict noncommutative domain $\mathcal{D}_{f,<}(\mathbb{C})$, we infer that

$$
\left\langle Z_{f, \varphi}^{\left(\lambda_{i}\right)}, Z_{f, \varphi}^{\left(\lambda_{j}\right)}\right\rangle_{f, \varphi}=\frac{\sqrt{1-\sum_{|\alpha| \geq 1} a_{\alpha}\left|\varphi_{\alpha}\left(\lambda_{j}\right)\right|^{2}} \sqrt{1-\sum_{|\alpha| \geq 1} a_{\alpha}\left|\varphi_{\alpha}\left(\lambda_{i}\right)\right|^{2}}}{1-\sum_{|\alpha| \geq 1} a_{\alpha}\left[\varphi\left(\lambda_{j}\right)\right]_{\alpha}\left[\overline{\varphi\left(\lambda_{i}\right)}\right]_{\alpha}} \neq 0
$$

for any $i, j=1, \ldots, k$. Hence, applying (4.7), (4.8), and (4.9), we conclude that $\Phi\left(\lambda_{j}\right)=A_{j}$, $j=1, \ldots, k$, if and only if $\left.\Phi\left(C_{1}, \ldots, C_{n}\right)^{*}\right|_{\mathcal{M} \otimes \mathcal{K}}=T^{*}$.

Since $\left\|\Phi\left(C_{1}, \ldots, C_{n}\right)\right\|=\|T\|$, it is clear that

$$
\left\|\Phi\left(C_{1}, \ldots, C_{n}\right)\right\| \leq 1 \quad \text { if and only if } \quad T T^{*} \leq I_{\mathcal{M} \otimes \mathcal{K}}
$$


On the other hand, for any $h_{1}, \ldots, h_{k} \in \mathcal{K}$, we have

$$
\begin{aligned}
& \left\langle\sum_{j=1}^{k} Z_{f, \varphi}^{\left(\lambda_{j}\right)} \otimes h_{j}, \sum_{j=1}^{k} Z_{f, \varphi}^{\left(\lambda_{j}\right)} \otimes h_{j}\right\rangle-\left\langle T^{*}\left(\sum_{j=1}^{k} Z_{f, \varphi}^{\left(\lambda_{j}\right)} \otimes h_{j}\right), T^{*}\left(\sum_{j=1}^{k} Z_{f, \varphi}^{\left(\lambda_{j}\right)} \otimes h_{j}\right)\right\rangle \\
& =\sum_{i, j=1}^{k}\left\langle Z_{f, \varphi}^{\left(\lambda_{i}\right)}, Z_{f, \varphi}^{\left(\lambda_{j}\right)}\right\rangle_{f, \varphi}\left\langle\left(I_{\mathcal{K}}-A_{j} A_{i}^{*}\right) h_{i}, h_{j}\right\rangle \\
& =\sum_{i, j=1}^{k} K_{f, \varphi}\left(\lambda_{j}, \lambda_{i}\right)\left\langle\left(I_{\mathcal{K}}-A_{j} A_{i}^{*}\right) h_{i}, h_{j}\right\rangle .
\end{aligned}
$$

Consequently, we deduce that $\left\|\Phi\left(C_{1}, \ldots, C_{n}\right)\right\| \leq 1$ if and only if matrix (4.3) is positive semidefinite. This completes the proof.

The following result is a noncommutative multivariable version of a result of Rosenblum and Rovnyak [31].

Theorem 4.3 Letf $:=\sum_{\alpha \in \mathbb{F}_{n}^{+}} a_{\alpha} Z_{\alpha}$ be a positive regular free holomorphic function, and let $\varphi=\left(\varphi_{1}, \ldots, \varphi_{n}\right)$ be an $n$-tuple offormal power series with model property. Let $\mathcal{I} \neq H^{\infty}\left(\mathbb{D}_{f, \varphi}\right)$ be a WOT-closed two-sided ideal of the noncommutative Hardy algebra $H^{\infty}\left(\mathbb{D}_{f, \varphi}\right)$. If $X \in$ $B\left(\mathcal{N}_{f, \varphi, \mathcal{I}} \otimes \mathcal{K}\right)$ is a self-adjoint operator, then the following statements are equivalent:

(i) $\Phi_{f, \varphi, B \otimes I_{\mathcal{K}}}(X) \leq X$, where $B \otimes I_{\mathcal{K}}:=\left(B_{1} \otimes I_{\mathcal{K}}, \ldots, B_{n} \otimes I_{\mathcal{K}}\right)$;

(ii) there are a Hilbert space $\mathcal{G}$ and a multi-analytic operator

$\Phi: \mathcal{N}_{f, \varphi, \mathcal{I}} \otimes \mathcal{G} \rightarrow \mathcal{N}_{f, \varphi, \mathcal{I}} \otimes \mathcal{K}$ with respect to the constrained weighted shifts $B_{1}, \ldots, B_{n}$ such that $X=\Phi \Phi^{*}$.

Proof First, we prove that (i) $\Rightarrow$ (ii). Since $\left(B_{1}, \ldots, B_{n}\right)$ is a pure $n$-tuple of operators in the noncommutative variety $\mathcal{V}_{f, \varphi, \mathcal{I}}\left(\mathcal{N}_{f, \varphi, \mathcal{I}}\right)$ and

$$
-\|X\| \Phi_{f, \varphi, B \otimes I_{\mathcal{K}}}^{m}(I) \leq \Phi_{f, \varphi, B \otimes I_{\mathcal{K}}}^{m}(X) \leq\|X\| \Phi_{f, \varphi, B \otimes I_{\mathcal{K}}}^{m}(I)
$$

we deduce that

$$
\text { SOT- } \lim _{m \rightarrow \infty} \Phi_{f, \varphi, B \otimes I_{\mathcal{K}}}^{m}(X)=0 \text {. }
$$

Notice that

$$
\Phi_{f, \varphi, B \otimes I_{\mathcal{K}}}^{m}(X) \leq \Phi_{f, \varphi, B \otimes I_{\mathcal{K}}}^{m-1}(X) \leq \cdots \leq X, \quad m \in \mathbb{N} .
$$

Then we obtain $X \geq 0$. Let $\mathcal{M}:=\overline{\operatorname{range} X^{\frac{1}{2}}}$ and define

$$
Q_{i}\left(X^{\frac{1}{2}} \xi\right):=X^{\frac{1}{2}}\left(\varphi_{i}(B)^{*} \otimes I_{\mathcal{K}}\right) \xi, \quad \xi \in \mathcal{N}_{f, \varphi, \mathcal{I}} \otimes \mathcal{K}
$$


for any $i=1, \ldots, n$. Note that

$$
\begin{aligned}
\sum_{|\alpha| \geq 1} a_{\alpha}\left\|Q_{\widetilde{\alpha}}\left(X^{\frac{1}{2}} \xi\right)\right\|^{2} & \leq \sum_{|\alpha| \geq 1}\left\|\sqrt{a_{\alpha}} X^{\frac{1}{2}}\left([\varphi(B)]_{\alpha}^{*} \otimes I_{\mathcal{K}}\right) \xi\right\|^{2} \\
& =\left\langle\Phi_{f, \varphi, B \otimes I_{\mathcal{K}}}(X) \xi, \xi\right\rangle \\
& \leq\left\|X^{\frac{1}{2}} \xi\right\|^{2}
\end{aligned}
$$

for any $\xi \in \mathcal{N}_{f, \varphi, \mathcal{I}} \otimes \mathcal{K}$. Hence, we obtain that

$$
a_{g_{i}}\left\|Q_{i} X^{\frac{1}{2}} \xi\right\|^{2} \leq\left\|X^{\frac{1}{2}} \xi\right\|^{2}, \quad \xi \in \mathcal{N}_{f, \varphi, \mathcal{I}} \otimes \mathcal{K},
$$

for any $i=1, \ldots, n$. Since $f$ is a positive regular free holomorphic function, each operator $Q_{i}, i=1, \ldots, n$, can be uniquely extended to a bounded operator (also denoted by $Q_{i}$ ) on $\mathcal{M}$. Denoting $A_{i}:=Q_{i}^{*}$ for any $i=1, \ldots, n$, we have

$$
\sum_{|\alpha| \geq 1} a_{\alpha} A_{\alpha} A_{\alpha}^{*} \leq I_{\mathcal{M}}
$$

where the convergence is in the weak operator topology. Setting $\phi_{A}(X):=\sum_{|\alpha| \geq 1} a_{\alpha} A_{\alpha} X A_{\alpha}^{*}$ (the convergence is in the weak operator topology) and using (4.10), we infer that

$$
\begin{aligned}
\left\langle\phi_{A}^{m}(I) X^{\frac{1}{2}} \xi, X^{\frac{1}{2}} \xi\right\rangle & =\left\langle\Phi_{f, \varphi, B \otimes I_{\mathcal{K}}}^{m}(X) \xi, \xi\right\rangle \\
& \leq\|X\|\left\langle\Phi_{f, \varphi, B \otimes I_{\mathcal{K}}}^{m}(I) \xi, \xi\right\rangle
\end{aligned}
$$

for any $\xi \in \mathcal{N}_{f, \varphi, \mathcal{I}} \otimes \mathcal{K}$, which implies that

$$
\text { SOT- } \lim _{m \rightarrow \infty} \phi_{A}^{m}(I)=0 .
$$

This shows that $A:=\left(A_{1}, \ldots, A_{n}\right)$ is a pure $n$-tuple of operators in $\mathcal{D}_{f}(\mathcal{M})$. According to Proposition 4.2 of [28], we know that $\mathcal{I}$ is a WOT-closed two-sided ideal of $H^{\infty}\left(\mathbb{D}_{f, \varphi}\right)$ if and only if there is a WOT-closed two-sided ideal $J$ of $F^{\infty}\left(\mathcal{D}_{f}\right)$ such that

$$
\mathcal{I}=\left\{\chi\left(\varphi\left(M_{Z}\right)\right): \chi \in J\right\}
$$

Taking into account that

$$
X^{\frac{1}{2}} A_{i}=\left(\varphi_{i}(B) \otimes I_{\mathcal{K}}\right) X^{\frac{1}{2}}, \quad i=1, \ldots, n
$$

Then, for any $\chi \in J$, we obtain

$$
X^{\frac{1}{2}} \chi\left(r A_{1}, \ldots, r A_{n}\right)=\left(\chi\left(r \varphi_{1}(B), \ldots, r \varphi_{n}(B)\right) \otimes I_{\mathcal{K}}\right) X^{\frac{1}{2}}
$$

for any $r \in(0,1)$. Moreover, since $\left(A_{1}, \ldots, A_{n}\right)$ is a pure $n$-tuple of operators in the noncommutative domain $\mathcal{D}_{f}(\mathcal{M})$ and $\left(\varphi_{1}(B), \ldots, \varphi_{n}(B)\right)$ is also a pure $n$-tuple of operators in $\mathcal{D}_{f}\left(\mathcal{N}_{f, \varphi, \mathcal{I}}\right)$, using $F^{\infty}\left(\mathcal{D}_{f}\right)$-functional calculus (see [24]), we have

$$
X^{\frac{1}{2}} \chi\left(A_{1}, \ldots, A_{n}\right)=\left(\chi\left(\varphi_{1}(B), \ldots, \varphi_{n}(B)\right) \otimes I_{\mathcal{K}}\right) X^{\frac{1}{2}}=0
$$


for any $\chi \in J$. Since $X^{\frac{1}{2}}$ is an injective operator on $\mathcal{M}$, we infer that

$$
\chi\left(A_{1}, \ldots, A_{n}\right)=0 \quad \text { for any } \chi \in J .
$$

Consequently, we deduce that $\left(A_{1}, \ldots, A_{n}\right)$ is a pure $n$-tuple of operators in the noncommutative variety $\mathcal{V}_{f, J}(\mathcal{M})$, where

$$
\mathcal{V}_{f, J}(\mathcal{M}):=\left\{\left(T_{1}, \ldots, T_{n}\right) \in \mathcal{D}_{f}(\mathcal{M}): \chi\left(T_{1}, \ldots, T_{n}\right)=0 \text { for any } \chi \in J\right\}
$$

Applying the appropriate result from [24], we know that the noncommutative Poisson kernel $K_{f, A}: \mathcal{M} \rightarrow \mathbb{H}_{f}^{2}(\varphi) \otimes \mathcal{G}(\mathcal{G}$ is an appropriate Hilbert space) defined by

$$
K_{f, A} h:=\sum_{\alpha \in \mathbb{F}_{n}^{+}} b_{\alpha} \varphi_{\alpha} \otimes \Delta_{f, A} A_{\alpha}^{*} h, \quad h \in \mathcal{M},
$$

where $\Delta_{f, A}:=\left(I-\sum_{|\alpha| \geq 1} a_{\alpha} A_{\alpha} A_{\alpha}^{*}\right)^{\frac{1}{2}}$ is an isometry with the properties that

$$
K_{f, A}(\mathcal{M}) \subseteq N_{f, \varphi, \mathcal{I}} \otimes \mathcal{G} \quad \text { and } \quad K_{f, A}^{*}\left(M_{\varphi_{i}} \otimes I_{\mathcal{G}}\right)=A_{i} K_{f, A}^{*}
$$

for any $i=1, \ldots, n$. Now we define

$$
\Phi:=X^{\frac{1}{2}} K_{f, A, \mathcal{I}}^{*}: \mathcal{N}_{f, \varphi, \mathcal{I}} \otimes \mathcal{G} \rightarrow \mathcal{N}_{f, \varphi, \mathcal{I}} \otimes \mathcal{K},
$$

where the constrained Poisson kernel $K_{f, A, \mathcal{I}}: \mathcal{M} \rightarrow \mathcal{N}_{f, \varphi, \mathcal{I}} \otimes \mathcal{G}$ is defined by

$$
K_{f, A, \mathcal{I}}:=\left(P_{\mathcal{N}_{f, \varphi, \mathcal{I}}} \otimes I_{\mathcal{G}}\right) K_{f, A}
$$

Since $\varphi$ has the model property, we have

$$
M_{\varphi_{i}}=\varphi_{i}\left(M_{Z_{1}}, \ldots, M_{Z_{n}}\right), \quad i=1, \ldots, n,
$$

where $\left(M_{Z_{1}}, \ldots, M_{Z_{n}}\right)$ is either in the $\operatorname{set} \mathcal{C}_{\varphi}^{\mathrm{SOT}}\left(\mathbb{H}_{f}^{2}(\varphi)\right)$ or $\mathcal{C}_{\varphi}^{\mathrm{rad}}\left(\mathbb{H}_{f}^{2}(\varphi)\right)$. Hence, we obtain

$$
K_{f, A, \mathcal{I}}^{*}\left(\varphi_{i}(B) \otimes I_{\mathcal{G}}\right)=A_{i} K_{f, A, \mathcal{I}}^{*}, \quad i=1, \ldots, n
$$

Therefore, using (4.11) and (4.12), we infer that

$$
\begin{aligned}
\Phi\left(\varphi_{i}(B) \otimes I_{\mathcal{G}}\right) & =X^{\frac{1}{2}} K_{f, A, \mathcal{I}}^{*}\left(\varphi_{i}(B) \otimes I_{\mathcal{G}}\right)=X^{\frac{1}{2}} A_{i} K_{f, A, \mathcal{I}}^{*} \\
& =\left(\varphi_{i}(B) \otimes I_{\mathcal{K}}\right) X^{\frac{1}{2}} K_{f, A, \mathcal{I}}^{*}=\left(\varphi_{i}(B) \otimes I_{\mathcal{K}}\right) \Phi
\end{aligned}
$$

for any $i=1, \ldots, n$. On the other hand, notice that

$$
\begin{aligned}
B_{i} & =\left.P_{\mathcal{N}_{f, \varphi}, \mathcal{I}} M_{Z_{i}}\right|_{\mathcal{N}_{f, \varphi, \mathcal{I}}} \\
& =\left.P_{\mathcal{N}_{f, \varphi, \mathcal{I}}} \psi_{i}\left(\varphi_{1}\left(M_{Z}\right), \ldots, \varphi_{n}\left(M_{Z}\right)\right)\right|_{\mathcal{N}_{f, \varphi, \mathcal{I}}} \\
& =\psi_{i}\left(\varphi_{1}(B), \ldots, \varphi_{n}(B)\right)
\end{aligned}
$$


for any $i=1, \ldots, n$. Then we conclude that each operator $B_{i}, i=1, \ldots, n$, is in the SOTclosure of all polynomials in $\varphi_{1}(B), \ldots, \varphi_{n}(B)$ and the identity. Consequently, we obtain that

$$
\Phi\left(B_{i} \otimes I_{\mathcal{G}}\right)=\left(B_{i} \otimes I_{\mathcal{K}}\right) \Phi, \quad i=1, \ldots, n .
$$

This shows that $\Phi$ is a multi-analytic operator with respect to the constrained weighted shifts $B_{1}, \ldots, B_{n}$. Moreover, since the constrained Poisson kernel $K_{f, A, \mathcal{I}}$ is an isometry, we deduce that

$$
\Phi \Phi^{*}=X^{\frac{1}{2}} K_{f, A, \mathcal{I}}^{*} K_{f, A, \mathcal{I}} X^{\frac{1}{2}}=X
$$

Now, we prove that (ii) $\Rightarrow$ (i). Note that $\left(B_{1}, \ldots, B_{n}\right) \in \mathcal{V}_{f, \varphi, \mathcal{I}}\left(\mathcal{N}_{f, \varphi, \mathcal{I}}\right)$. Then we have

$$
\begin{aligned}
\Phi_{f, \varphi, B \otimes I_{\mathcal{K}}}(X) & =\sum_{|\alpha| \geq 1} a_{\alpha}\left([\varphi(B)]_{\alpha} \otimes I_{\mathcal{K}}\right) X\left([\varphi(B)]_{\alpha} \otimes I_{\mathcal{K}}\right)^{*} \\
& =\sum_{|\alpha| \geq 1} a_{\alpha}\left([\varphi(B)]_{\alpha} \otimes I_{\mathcal{K}}\right) \Phi \Phi^{*}\left([\varphi(B)]_{\alpha} \otimes I_{\mathcal{K}}\right)^{*} \\
& =\Phi\left(\sum_{|\alpha| \geq 1} a_{\alpha}\left([\varphi(B)]_{\alpha} \otimes I_{\mathcal{G}}\right)\left([\varphi(B)]_{\alpha} \otimes I_{\mathcal{G}}\right)^{*}\right) \Phi^{*} \\
& \leq \Phi \Phi^{*}=X
\end{aligned}
$$

where the convergence is in the weak operator topology. This completes the proof.

As an application, we obtain a Beurling-type characterization of the invariant subspaces under the constrained weighted shifts $B_{1}, \ldots, B_{n}$.

Theorem 4.4 Letf $:=\sum_{\alpha \in \mathbb{F}_{n}^{+}} a_{\alpha} Z_{\alpha}$ be a positive regular free holomorphic function, and let $\varphi=\left(\varphi_{1}, \ldots, \varphi_{n}\right)$ be an n-tuple of formal power series with model property. Let $\mathcal{I} \neq H^{\infty}\left(\mathbb{D}_{f, \varphi}\right)$ be a WOT-closed two-sided ideal of the noncommutative Hardy algebra $H^{\infty}\left(\mathbb{D}_{f, \varphi}\right)$. A subspace $\mathcal{M} \subseteq \mathcal{N}_{f, \varphi, \mathcal{I}} \otimes \mathcal{K}$ is invariant under $B_{i} \otimes I_{\mathcal{K}}, i=1, \ldots, n$, if and only if there are $a$ Hilbert space $\mathcal{G}$ and an inner multi-analytic operator

$$
\Phi: \mathcal{N}_{f, \varphi, \mathcal{I}} \otimes \mathcal{G} \rightarrow \mathcal{N}_{f, \varphi, \mathcal{I}} \otimes \mathcal{K}
$$

with respect to the constrained weighted shifts $B_{1}, \ldots, B_{n}$ such that

$$
\mathcal{M}=\Phi\left[\mathcal{N}_{f, \varphi, \mathcal{I}} \otimes \mathcal{G}\right]
$$

Proof First, we assume that $\mathcal{M} \subseteq \mathcal{N}_{f, \varphi, \mathcal{I}} \otimes \mathcal{K}$ is invariant under $B_{1} \otimes I_{\mathcal{K}}, \ldots, B_{n} \otimes I_{\mathcal{K}}$. Notice that

$$
P_{\mathcal{M}}\left(B_{i} \otimes I_{\mathcal{K}}\right) P_{\mathcal{M}}=\left(B_{i} \otimes I_{\mathcal{K}}\right) P_{\mathcal{M}}, \quad i=1, \ldots, n,
$$


and $\left(B_{1}, \ldots, B_{n}\right) \in \mathcal{V}_{f, \varphi, \mathcal{I}}\left(\mathcal{N}_{f, \varphi, \mathcal{I}}\right)$. Then we have

$$
\begin{aligned}
\Phi_{f, \varphi, B \otimes I_{\mathcal{K}}}\left(P_{\mathcal{M}}\right) & =P_{\mathcal{M}}\left(\sum_{|\alpha| \geq 1} a_{\alpha}\left([\varphi(B)]_{\alpha} \otimes I_{\mathcal{K}}\right) P_{\mathcal{M}}\left([\varphi(B)]_{\alpha}^{*} \otimes I_{\mathcal{K}}\right)\right) P_{\mathcal{M}} \\
& \leq P_{\mathcal{M}}\left(\sum_{|\alpha| \geq 1} a_{\alpha}\left([\varphi(B)]_{\alpha} \otimes I_{\mathcal{K}}\right)\left([\varphi(B)]_{\alpha}^{*} \otimes I_{\mathcal{K}}\right)\right) P_{\mathcal{M}} \\
& =P_{\mathcal{M}}\left(\sum_{|\alpha| \geq 1} a_{\alpha}[\varphi(B)]_{\alpha}[\varphi(B)]_{\alpha}^{*} \otimes I_{\mathcal{K}}\right) P_{\mathcal{M}} \\
& \leq P_{\mathcal{M}} .
\end{aligned}
$$

According to Theorem 4.3, there are a Hilbert space $\mathcal{G}$ and a multi-analytic operator

$$
\Phi: \mathcal{N}_{f, \varphi, \mathcal{I}} \otimes \mathcal{G} \rightarrow \mathcal{N}_{f, \varphi, \mathcal{I}} \otimes \mathcal{K}
$$

with respect to the constrained weighted shifts $B_{1}, \ldots, B_{n}$ such that $P_{\mathcal{M}}=\Phi \Phi^{*}$. Moreover, since $P_{\mathcal{M}}$ is an orthogonal projection, we deduce that $\Phi$ is a partial isometry and $\mathcal{M}=$ $\Phi\left[\mathcal{N}_{f, \varphi, \mathcal{I}} \otimes \mathcal{G}\right]$. The converse is obvious. This completes the proof.

\section{Acknowledgements}

The authors wish to thank the anonymous referees and the editor for their useful comments.

\section{Funding}

This work is supported by the National Natural Science Foundation of China (No. 11771340).

\section{Availability of data and materials}

Not applicable.

\section{Competing interests}

The authors declare that they have no competing interests.

\section{Authors' contributions}

The authors contributed equally. All authors read and approved the final manuscript.

\section{Author details}

'School of Mathematics and Statistics, Hubei Normal University, Huangshi, China. ${ }^{2}$ School of Mathematics and Statistics, Wuhan University, Wuhan, China.

\section{Publisher's Note}

Springer Nature remains neutral with regard to jurisdictional claims in published maps and institutional affiliations.

Received: 18 October 2019 Accepted: 14 May 2020 Published online: 25 May 2020

\section{References}

1. Ball, J., Vinnikov, V.: Lax-Phillips Scattering and Conservative Linear Systems: A Cuntz-Algebra Multidimensional Setting. Mem. Amer. Math. Soc., vol. 837 (2005)

2. Bunce, J.: Models for $n$-tuples of noncommuting operators. J. Funct. Anal. 57, 21-30 (1984)

3. Davidson, K., Pitts, D.: Nevanlinna-Pick interpolation for noncommutative analytic Toeplitz algebras. Integral Equ. Oper. Theory 31, 321-337 (1998)

4. Davidson, K., Pitts, D.: The algebraic structure of non-commutative analytic Toeplitz algebras. Math. Ann. 311 , 275-303 (1998)

5. Foiaş, C.: On Hille's spectral theory and operational calculus for semi-groups of operators in Hilbert space. Compos. Math. 14, 71-73 (1959)

6. Foiaş, C., Frazho, A.: The Commutant Lifting Approach to Interpolation Problems. Operator Theory: Advances and Applications. Birhäuser, Bassel (1990)

7. Foiaş, C., Frazho, A., Gohberg, I., Kaashoek, M.: Metric Constrained Interpolation, Commutant Lifting and Systems. Operator Theory: Advances and Applications, vol. 100. Birhäuser, Bassel (1998)

8. Frazho, A.: Models for noncommuting operators. J. Funct. Anal. 48, 1-11 (1982)

9. Helton, J.: "Positive" noncommutative polynomials are sums of squares. Ann. Math. (2) 156(2), 675-694 (2002) 
10. Helton, J., McCullough, S., Vinnikov, V.: Noncommutative convexity arises from linear matrix inequalities. J. Funct. Anal. 240(1), 105-191 (2006)

11. Hu, J., Wang, M.: Free holomorphic functions on the noncommutative polydomains and universal models. Results Math. 73, Article ID 99 (2018)

12. Muhly, P., Solel, B.: Tensor algebras over $C^{*}$-correspondences: representations, dilations, and $C^{*}$-envelopes. J. Funct. Anal. 158, 389-457 (1998)

13. Muhly, P., Solel, B.: Hardy algebras, W*-correspondences and interpolation theory. Math. Ann. 330, 353-415 (2004)

14. Muhly, P., Solel, B.: Canonical models for representations of Hardy algebras. Integral Equ. Oper. Theory 53, 411-452 (2005)

15. Pisier, G.: Similarity Problems and Completely Bounded Maps. Lect. Notes Math., vol. 1618. Springer, New York (1995)

16. Popescu, G.: Isometric dilations for infinite sequences of noncommuting operators. Trans. Am. Math. Soc. 316 523-536 (1989)

17. Popescu, G.: Multi-analytic operators and some factorization theorems. Indiana Univ. Math. J. 38, 693-710 (1989)

18. Popescu, G.: Von Neumann inequality for $\left(B(H)^{n}\right)_{1}$. Math. Scand. 68, 292-304 (1991)

19. Popescu, G.: Multi-analytic operators on Fock spaces. Math. Ann. 303, 31-46 (1995)

20. Popescu, G.: Poisson transforms on some $C^{*}$-algebras generated by isometries. J. Funct. Anal. 161, 27-61 (1999)

21. Popescu, G.: Entropy and Multivariable Interpolation. Mem. Amer. Math. Soc., vol. 868 (2006)

22. Popescu, G.: Free holomorphic functions on the unit ball of $B(\mathcal{H})^{n}$. J. Funct. Anal. 241, 268-333 (2006)

23. Popescu, G.: Noncommutative Berezin transforms and multivariable operator model theory. J. Funct. Anal. 254(4), 1003-1057 (2008)

24. Popescu, G.: Operator Theory on Noncommutative Domains. Mem. Amer. Math. Soc., vol. 964 (2010)

25. Popescu, G.: Free biholomorphic functions and operator model theory. J. Funct. Anal. 262, 3240-3308 (2012)

26. Popescu, G.: Free biholomorphic functions and operator model theory, II. J. Funct. Anal. 265, 786-836 (2013)

27. Popescu, G.: Berezin transforms on noncommutative varieties in polydomains. J. Funct. Anal. 265(10), 2500-2552 (2013)

28. Popescu, G.: Noncommutative multivariable operator theory. Integral Equ. Oper. Theory 75, 87-133 (2013)

29. Popescu, G.: Curvature invariant on noncommutative polyballs. Adv. Math. 279, 104-158 (2015)

30. Popescu, G.: Berezin transforms on noncommutative polydomains. Trans. Am. Math. Soc. 368, 4357-4416 (2016)

31. Rosenblum, M., Rovnyak, J.: Hardy Classes and Operator Theory. Oxford University Press, New York (1985)

32. Sarason, D.: Generalized interpolation in $H^{\infty}$. Trans. Am. Math. Soc. 127, 179-203 (1967)

33. Sz.-Nagy, B., Foias, C.: Harmonic Analysis of Operators on Hilbert Space. North-Holland, Amsterdam (1970)

34. Wang, M., Hu, J.: Free holomorphic functions on the regular polyball. Complex Anal. Oper. Theory 12(7), 1617-1635 (2018)

\section{Submit your manuscript to a SpringerOpen ${ }^{\circ}$ journal and benefit from:}

- Convenient online submission

- Rigorous peer review

- Open access: articles freely available online

- High visibility within the field

- Retaining the copyright to your article

Submit your next manuscript at $\gg$ springeropen.com 